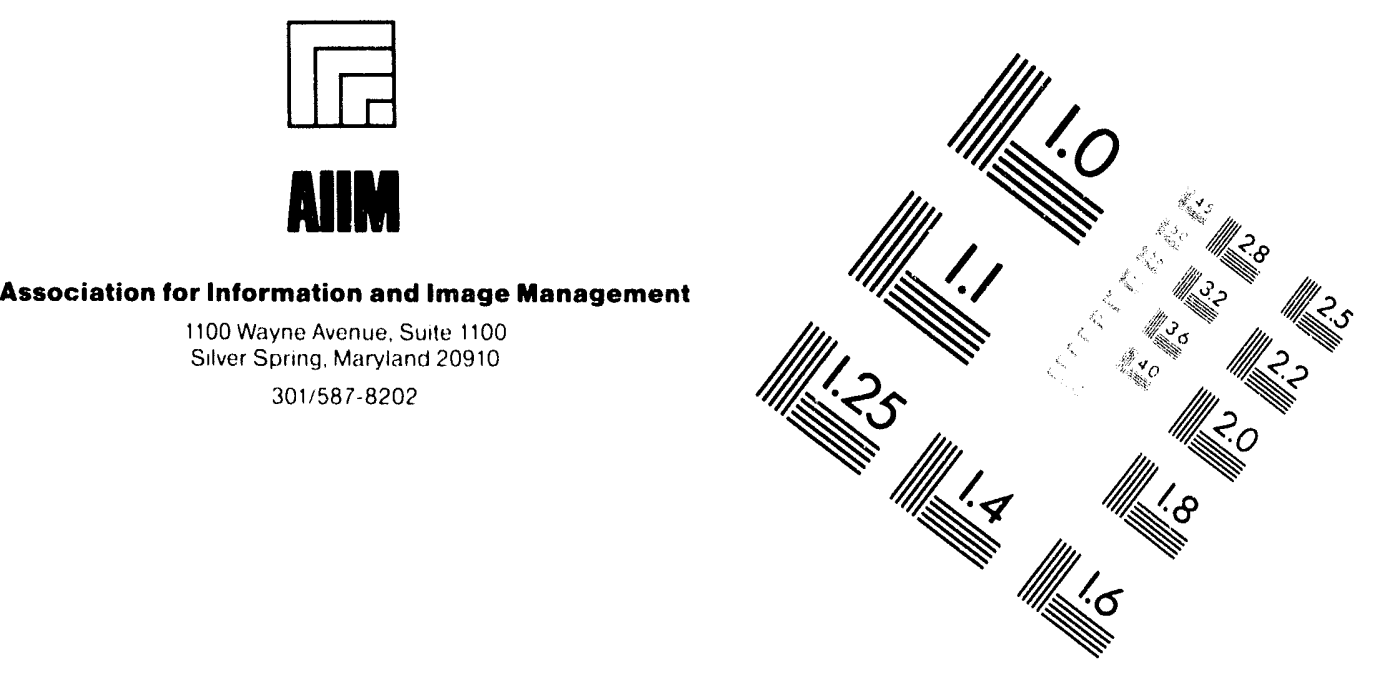

\title{
Centimeter
}

$\begin{array}{llllllllllllllll}1 & 2 & 3 & 4 & 5 & 6 & 7 & 8 & 9 & 10 & 11 & 12 & 13 & 14 & 15 & \mathrm{~mm}\end{array}$

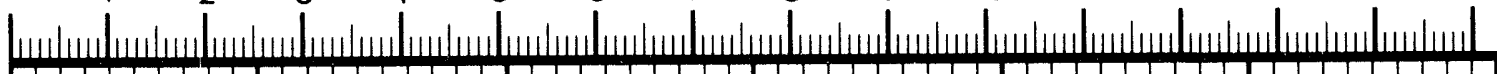
Inches
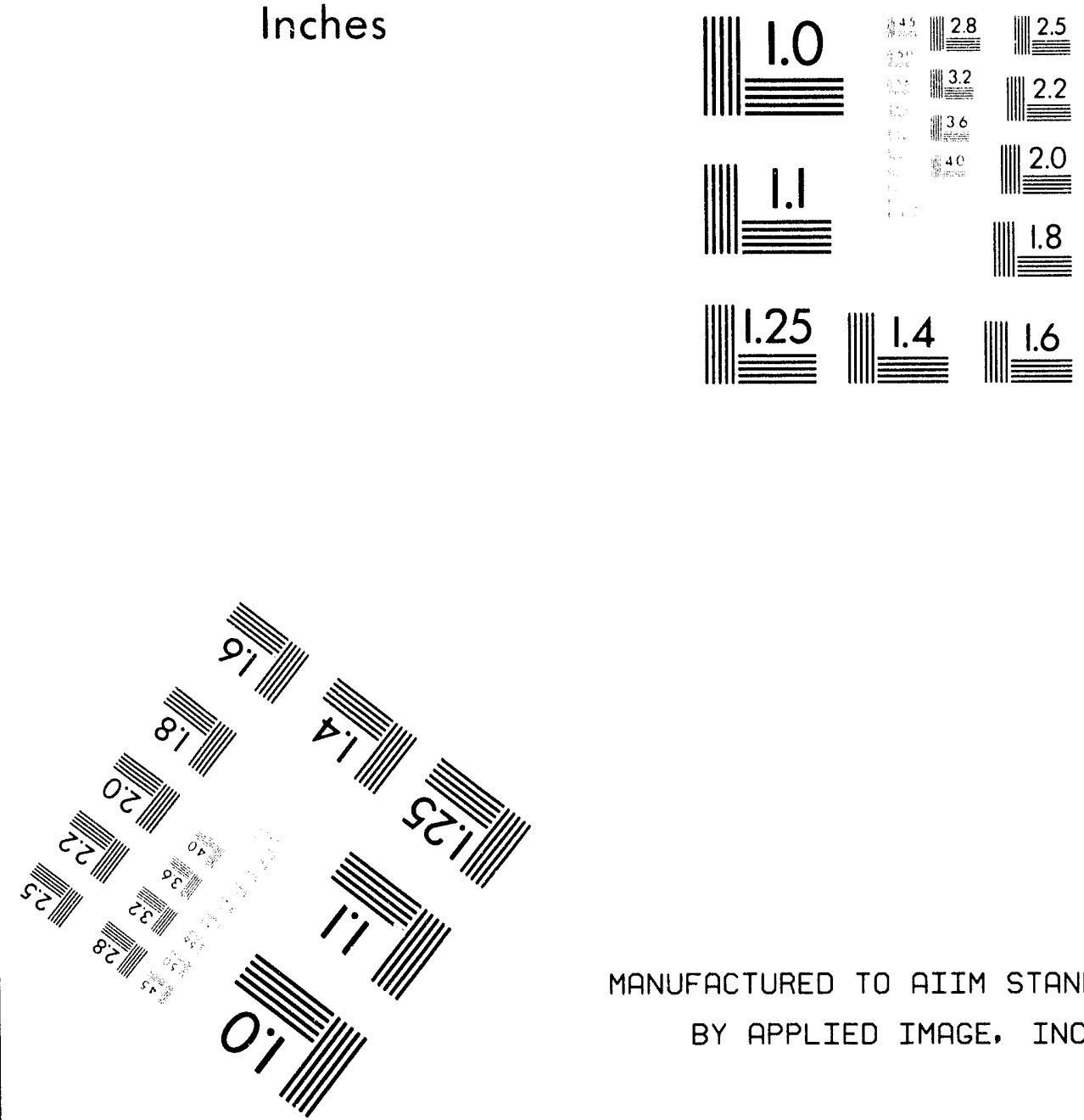

MANUFACTURED TO AIIM STANDARDS

BY APPLIED IMAGE, INC.

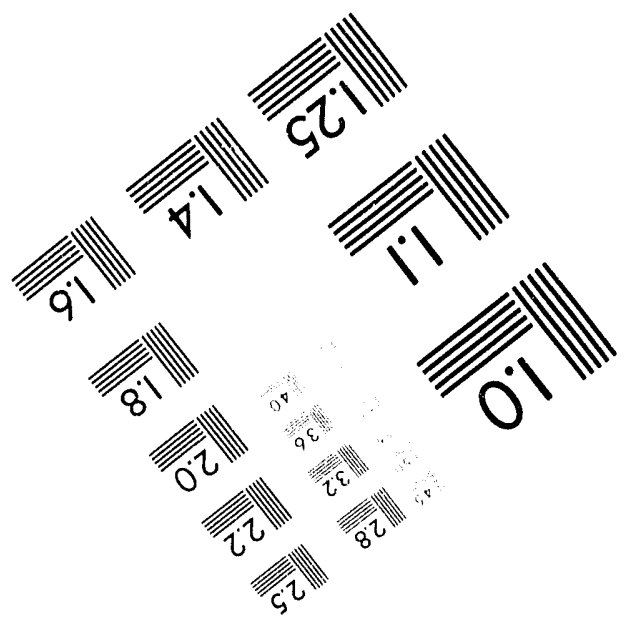



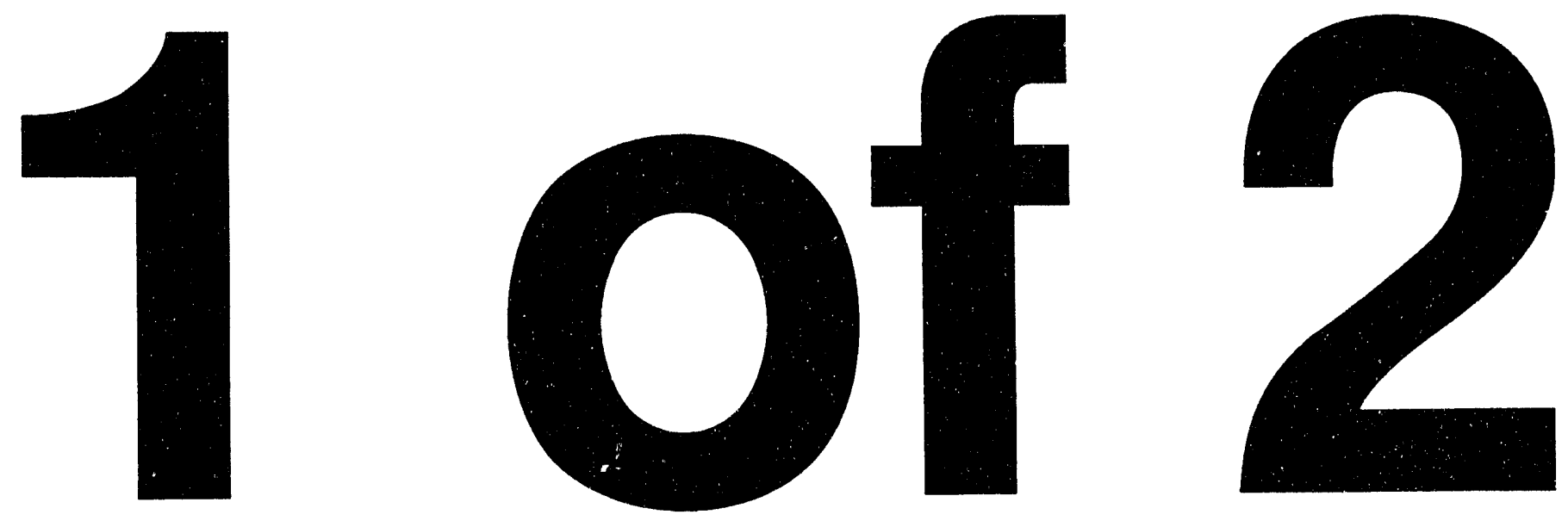


\section{User Instructions for the CIDER Dose Code}

Hanford Environmental Dose Reconstruction Project

P. W. Eslinger

K. S. Lessor

S. J. Ouderkirk

May 1994

Prepared for the Technical Steering Panel and the Centers for Disease Control and Prevention under Contract 200-92-0503(CDC)/18620(BNW)

\section{Battelle}

Pacific Northwest Laboratories

Richland, Washington 99352 


\section{Preface}

In 1987, the U.S. Department of Energy (DOE) directed the Pacific Northwest Laboratory, which is operated by Battelle Memorial Institute, to conduct the Hanford Environmental Dose Reconstruction (HEDR) Project. The DOE directive to begin project work followed a recommendation by the Hanford Health Effects Review (HHER) Panel in 1986. The HHER Panel was formed to consider the potential health implications of past Hanford-Site releases of radioactive materials.

Members of a Technical Steering Panel (TSP) were selected to direct the HEDR Project work. The TSP consists of experts in the various technical fields relevant to HEDR Project work and representatives from the states of Washington, Oregon, and Idaho; Native American Tribes; and the public. The technical members on the panel were selected by the vice presidents for research at major universities in Washington and Oregon. The state representatives were selected by the respective state governments. The Native American tribes and public representatives were selected by the other panel members.

A December 1990 memorandum of understanding between the Secretaries of the DOE and the U.S. Department of Health and Human Services (DHHS) transferred responsibility for managing the DOE's dose reconstruction and exposure assessment studies to the DHHS. This transfer resulted in the current contract between Battelle, Pacific Northwest Laboratories (BNW) and the Centers for Disease Control and Prevention, an agency of the DHHS, to continue the project.

The purpose of the HEDR Project is to estimate the radiation dose that individuals could have received as a result of emissions since 1944 from the Hanford Site near Richland, Washington. The HEDR Project work is conducted under several technical and administrative tasks, among which is the Environmental Pathways and Dose Estimates task. The staff on this task have developed a suite of computer codes which are used to estimate doses to individuals of the public.

The purpose of this document is to provide user instructions for the CIDER (Calculation of Individual Doses from Environmental Radionuclides) computer code. The document also provides user instructions for several utility codes used to build input data libraries for CIDER. The CIDER code computes annual doses for both reference individuals and individuals with a known residence and food consumption history. 


\section{Summary}

\section{Scope of Work}

This document prov des user instructions for the CIDER (Calculation of Individual Doses from Environmental Radionuc. ides) computer code. The CIDER code computes estimates of annual doses estimated for both reference individuals and individuals with a known residence and food consumption history.

This document also provides user instructions for four utility codes used to build input data libraries for CIDER. These utility codes are ENVFAC (environmental factors), FOOFAC (food factors), LIFFAC (lifestyle factors), and ORGFAC (organ factors).

Finally, this document provides user instructions for the EXPAND utility code. The EXPAND code processes a result file from CIDER and extracts a summary of the dose information for reporting or plotting purposes.

\section{Approach to Modeling Uncertainties}

The suite of codes (including source term calculations, air transport modeling, and environmental concentration estimation) for modeling releases of ${ }^{131}$ I through air pathways were designed to provide estimates of uncertainty in the dose estimates. The approach chosen was to provide 100 dose estimates rather than a single number. Each dose estimate utilizes a different set of input values for hundreds of random variables (for which values are not known with certainty). Some examples of random variables are wind speed and direction, frost dates, plant growth parameters, animal eating habits, food distribution networks, and dose conversion factors. Each specific set (or realization) of input variables produces a single output estimate (also called a realization) of dose.

\section{Data Requirements}

The CIDER code relies on a database of environmental concentrations of ${ }^{131}$ I produced by other HEDR computer codes. The database is produce by the DESCARTES (Dynamic Estimates of Concentrations and $\underline{A}$ ccumulated $\underline{R}$ adionuclides in Terrestrial Environments) code. The CIDER code relies on this 1-gigabyte database as well as some related data libraries containing realizations of random parameters in order to produce dose estimates. 


\section{Glossary}

AGGREGATE the code that translates the output from DESCARTES to the form required by CIDER and imposes a minimum dose filter on the data

CIDER

the code for estimating annual dose to humans (Calculation of Individual Doses from Environmental Radionuclides)

DESCARTES the code for estimating environmental contaminants (Dynamic Estimates of Concentrations and Accumulated Radionuclides in Terrestrial Environments)

double

an eight-byte real value

HEDR

the Hanford Environmental Dose Reconstruction project

HEDRIC

the HEDR Integrated Codes

integer

a four-byte signed integer value

RATCHET

the code for air radionuclide transport Regional Atmospheric Transport Code for Hanford Emission Tracking)

real

a four-byte real value

realization

one of a set of code input values; or a code output result that is based on one of a set of code input values (For example, a single dose estimate produced by CIDER is one realization based on a specific realization of input variables.)

record

a block of data in a file, consisting of one or more tokens occupying one or more lines and terminated by a semicolon (;)

token

a string of characters consisting of alphanumeric characters plus the following characters: underscore $U$, minus $(-)$, plus $(+)$, backslash () , forward slash $(I)$, decimal (.), and colon (:) - No white space (blank, tab, line feed) is allowed within the token. 


\section{Contents}

Preface $\ldots \ldots \ldots \ldots \ldots \ldots \ldots \ldots \ldots \ldots \ldots \ldots \ldots \ldots \ldots \ldots \ldots$

Summary $\ldots \ldots \ldots \ldots \ldots \ldots \ldots \ldots \ldots \ldots \ldots \ldots \ldots \ldots \ldots$

Glossary $\ldots \ldots \ldots \ldots \ldots \ldots \ldots \ldots \ldots \ldots \ldots \ldots \ldots \ldots \ldots \ldots$ vii

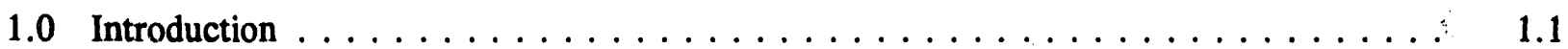

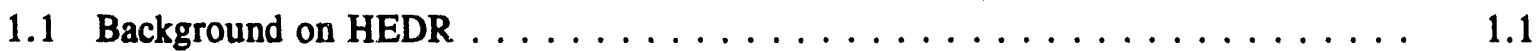

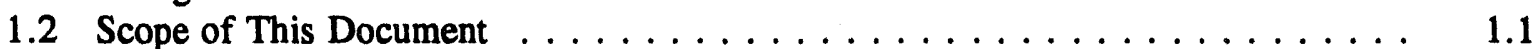

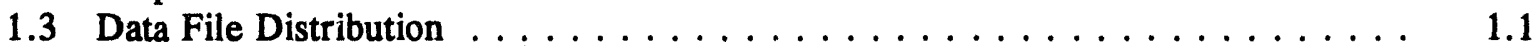

1.4 Node Locations . . . . . . . . . . . . . . . . . . . . . . . . . 1.2

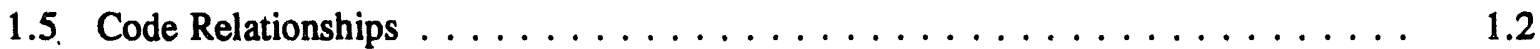

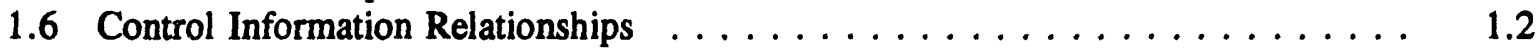

1.7 Computing Environment $\ldots \ldots \ldots \ldots \ldots \ldots \ldots \ldots \ldots \ldots \ldots$

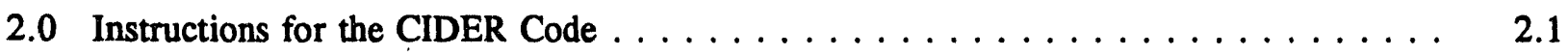

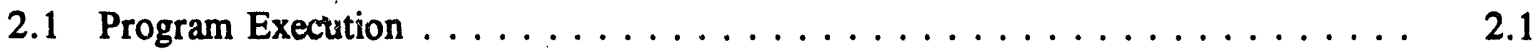

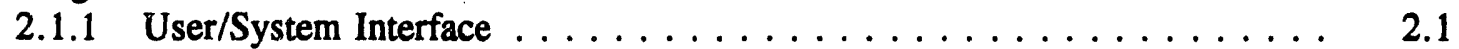

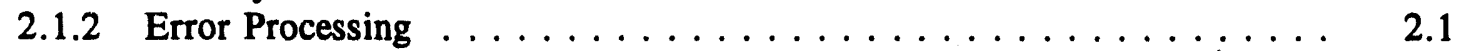

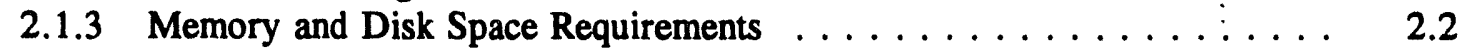

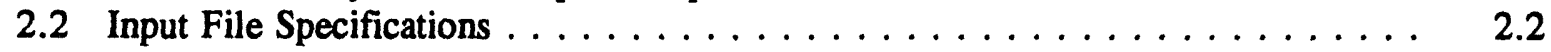

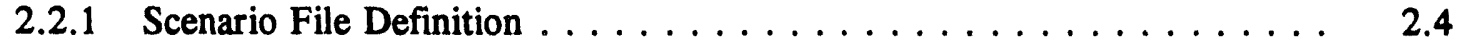

2.2 .2 Node Concentration Files . . . . . . . . . . . . . . 2.14

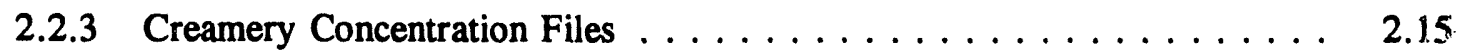

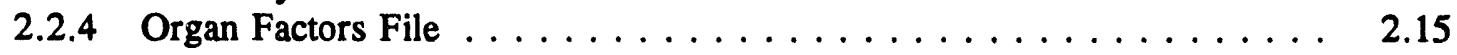

2.2.5 Node Index and Creamery Index Files . . . . . . . . . . . 2.15

2.2 .6 Reference Diet File . . . . . . . . . . . . . . . . . 2.16

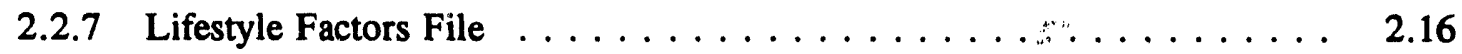

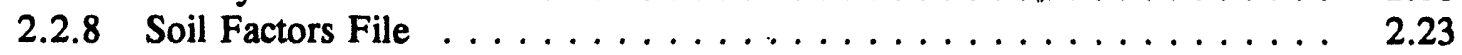

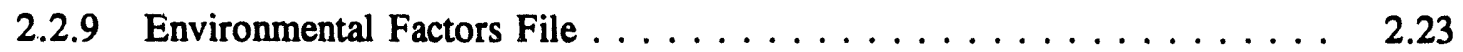

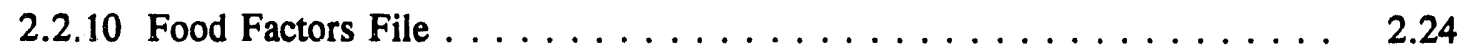

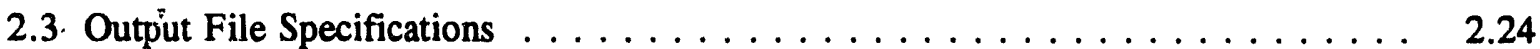

2.3.1 Result File . . . . . . . . . . . . . . . . . . . . . . . 2.24

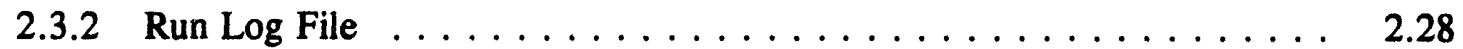

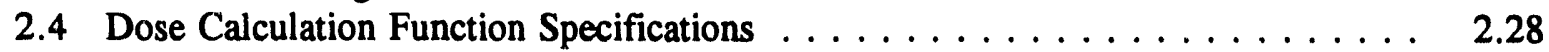

2.4.1 External Dose . . . . . . . . . . . . . . . . . . 2.29

2.4 .2 Inhalation Dose . . . . . . . . . . . . . . . . . . . . 2.30

2.4.3 Ingestion Dose from Single-Compartment Foods . . . . . . . . . . . . 2.30

2.4.4 Ingestion Dose from Animal Products . . . . . . . . . . . . . . 2.31

2.4.5 Ingestion Dose from Double-Compartment Foods . . . . . . . . . . . 2.32

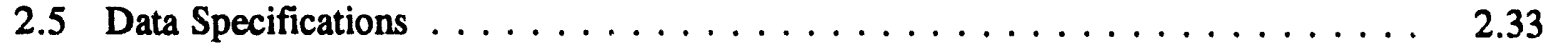

2.5.1 CIDER Data Dictionary . . . . . . . . . . . . . . 2.33 
2.5.2 Structure Definitions . . . . . . . . . . . . . . . . 2.35

2.5.3 Parameter Range Definitions . . . . . . . . . . . . . . 2.36

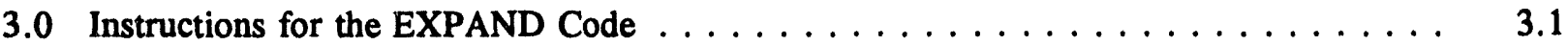

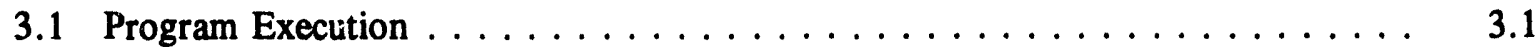

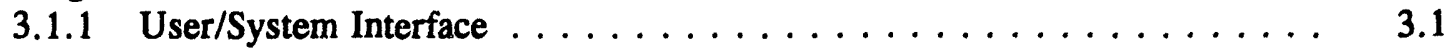

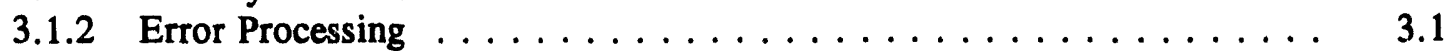

3.1.3 Memory and Disk Space Requirements $\ldots \ldots \ldots \ldots \ldots \ldots \ldots \ldots . \ldots \ldots$

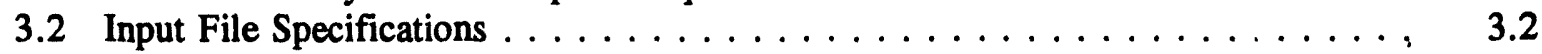

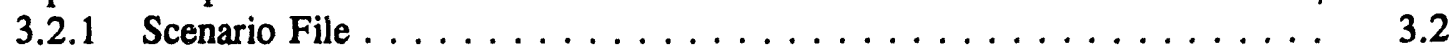

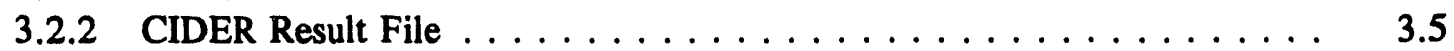

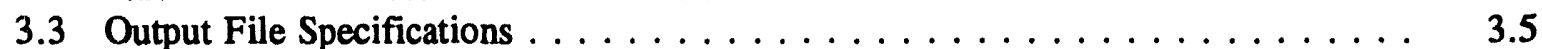

3.3.1 EXPAND Result File $\ldots \ldots \ldots \ldots \ldots \ldots \ldots \ldots \ldots \ldots$

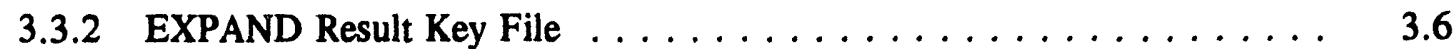

3.3.3 Run Log File . . . . . . . . . . . . . . . . . . . . . . . 3.7

3.4 Algorithm Specifications $\ldots \ldots \ldots \ldots \ldots \ldots \ldots \ldots \ldots \ldots \ldots$

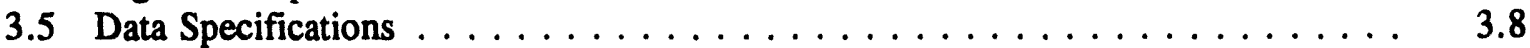

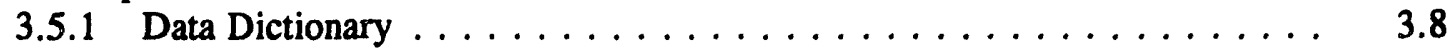

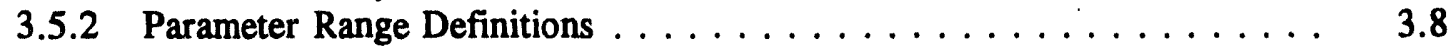

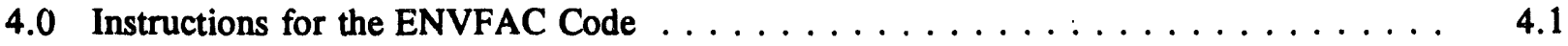

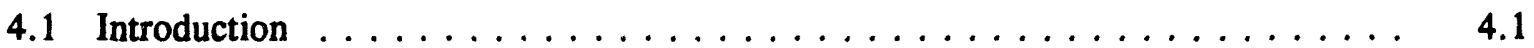

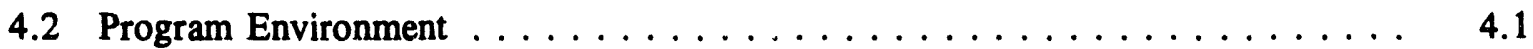

4.2.1 User/System Interface . . . . . . . . . . . . . . . . 4.1

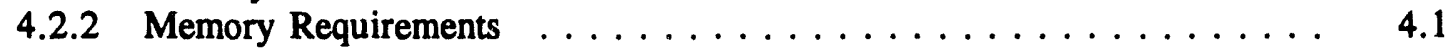

4.2.3 Data Size Limitations $\ldots \ldots \ldots \ldots \ldots \ldots \ldots \ldots \ldots \ldots \ldots . \ldots \ldots . \ldots \ldots$

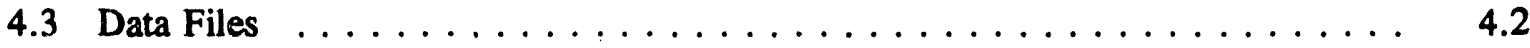

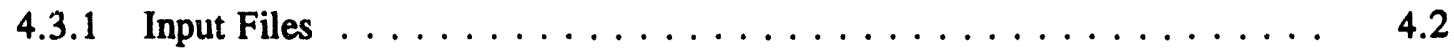

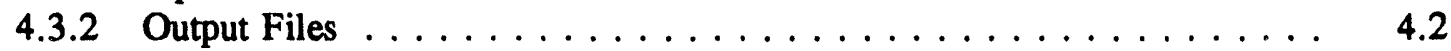

4.4 ENVFAC Keyword Command Descriptions $\ldots \ldots \ldots \ldots \ldots \ldots \ldots \ldots$

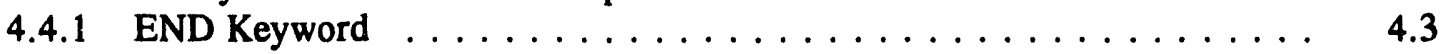

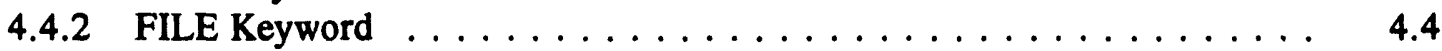

4.4 .3 NUCLIDE Keyword $\ldots \ldots \ldots \ldots \ldots \ldots \ldots \ldots \ldots \ldots \ldots \ldots$

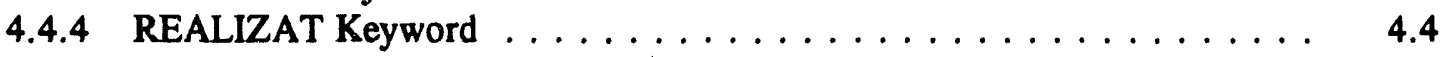

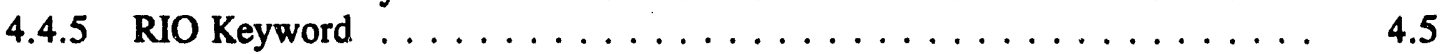

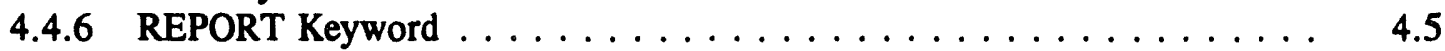

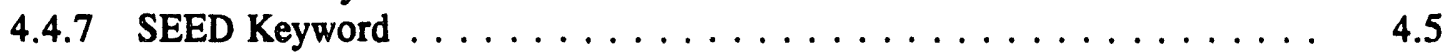

4.4 .8 SHIELD Keyword $\ldots \ldots \ldots \ldots \ldots \ldots \ldots \ldots \ldots \ldots \ldots$

4.4 .9 TITLE Keyword $\ldots \ldots \ldots \ldots \ldots \ldots \ldots \ldots \ldots \ldots$

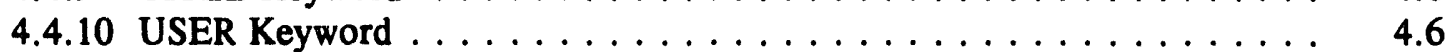

4.5 Example Files for the ENVFAC Code $\ldots \ldots \ldots \ldots \ldots \ldots \ldots \ldots \ldots$

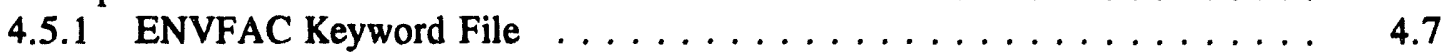

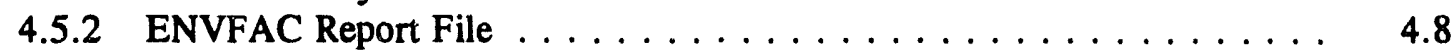

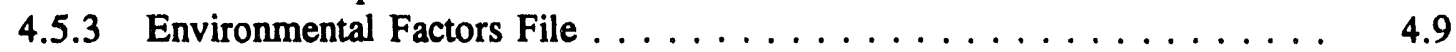

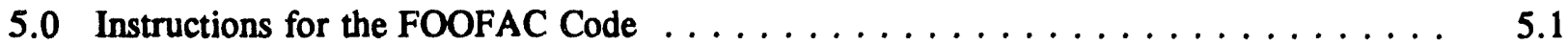

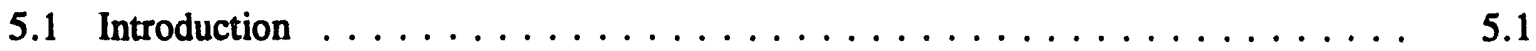




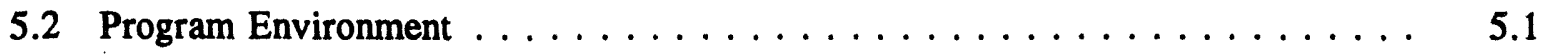

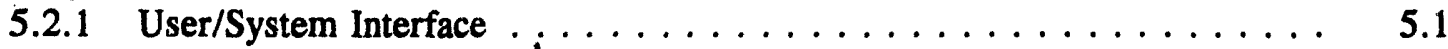

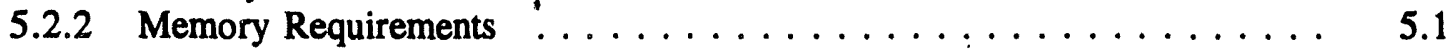

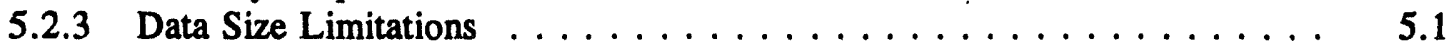

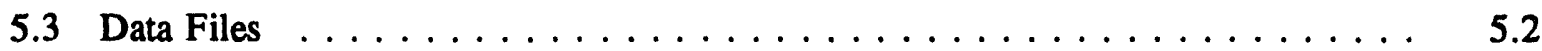

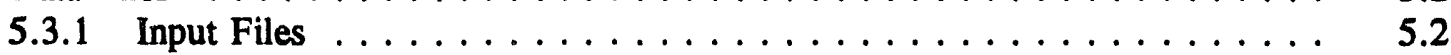

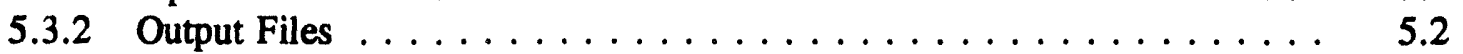

5.4 FOOFAC Keyword Command Descriptions $\ldots \ldots \ldots \ldots \ldots \ldots$

5.4 .1 DISTR Keyword $\ldots \ldots \ldots \ldots \ldots \ldots \ldots \ldots \ldots \ldots \ldots$

5.4 .2 DRYWET Keyword $\ldots \ldots \ldots \ldots \ldots \ldots \ldots \ldots \ldots \ldots \ldots$

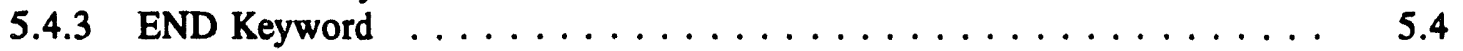

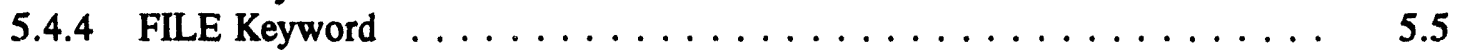

5.4 .5 FOOD Keyword $\ldots \ldots \ldots \ldots \ldots \ldots \ldots \ldots \ldots \ldots \ldots \ldots \ldots$

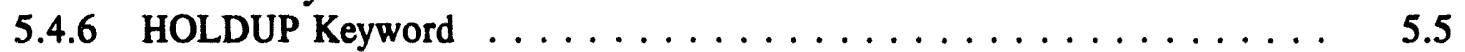

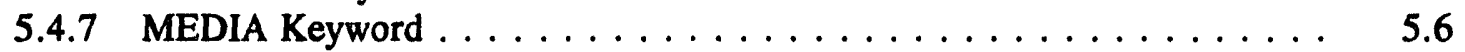

5.4 .8 NUCLIDE Keyword $\ldots \ldots \ldots \ldots \ldots \ldots \ldots \ldots \ldots \ldots$

5.4 .9 REALIZAT Keyword $\ldots \ldots \ldots \ldots \ldots \ldots \ldots \ldots \ldots \ldots$

5.4 .10 REPORT Keyword $\ldots \ldots \ldots \ldots \ldots \ldots \ldots \ldots \ldots . \ldots \ldots$

5.4 .11 RETAIN Keyword $\ldots \ldots \ldots \ldots \ldots \ldots \ldots \ldots \ldots \ldots \ldots$

5.4 .12 SEED KEYWORD $\ldots \ldots \ldots \ldots \ldots \ldots \ldots \ldots \ldots \ldots$

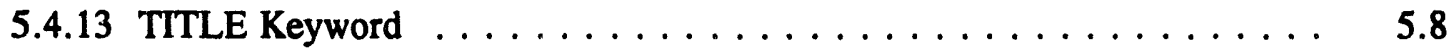

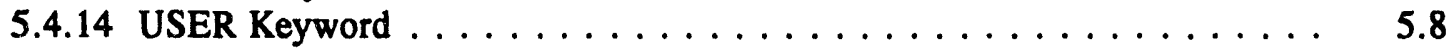

5.5 Example Files for the FOOFAC Code $\ldots \ldots \ldots \ldots \ldots \ldots$

5.5 .1 FOOFAC Keyword File $\ldots \ldots \ldots \ldots \ldots \ldots \ldots \ldots$

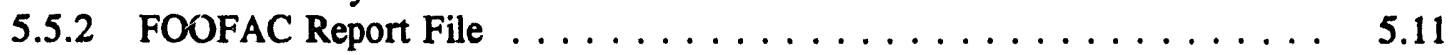

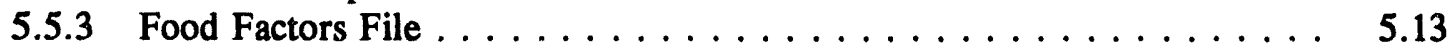

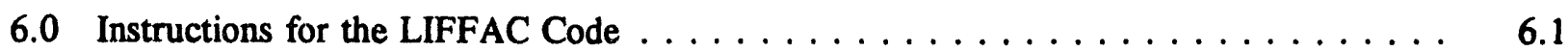

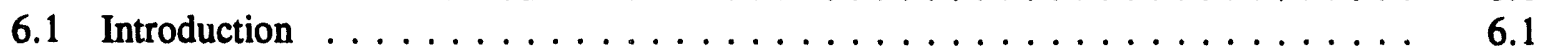

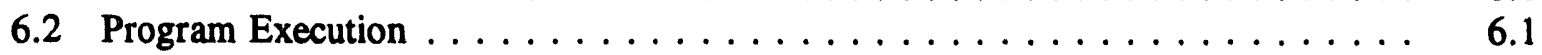

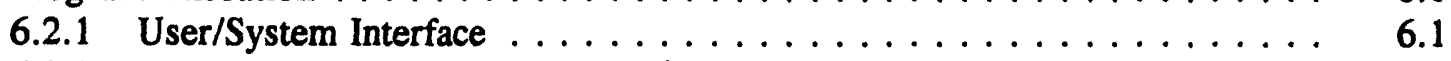

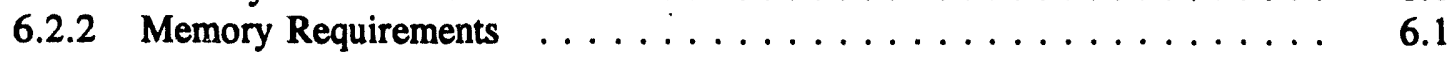

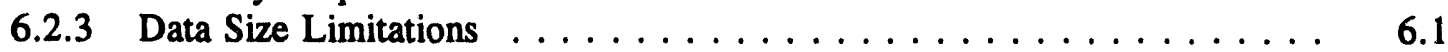

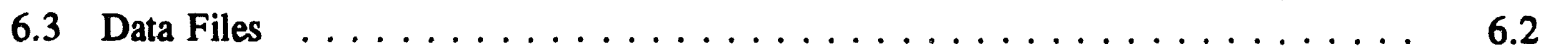

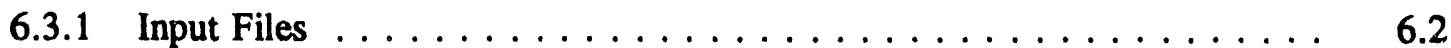

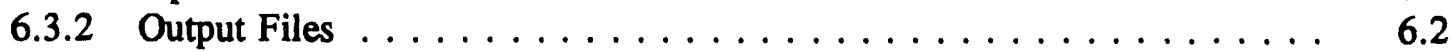

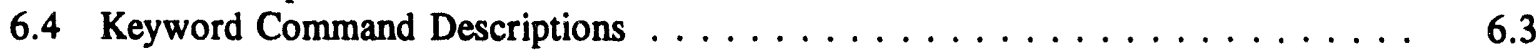

6.4 AGEBR Keyword $\ldots \ldots \ldots \ldots \ldots \ldots \ldots \ldots \ldots \ldots . \ldots \ldots \ldots$

6.4 .2 AGEFO Keyword $\ldots \ldots \ldots \ldots \ldots \ldots \ldots \ldots \ldots \ldots \ldots$

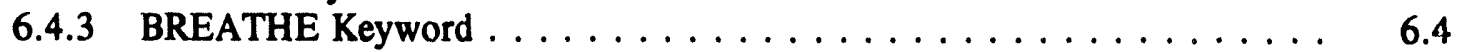

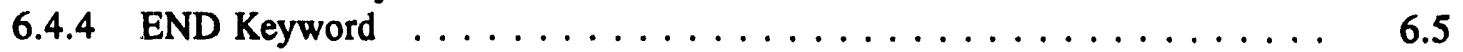

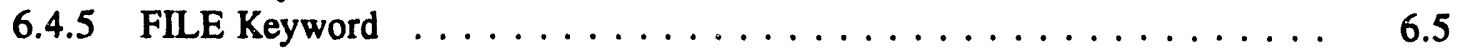

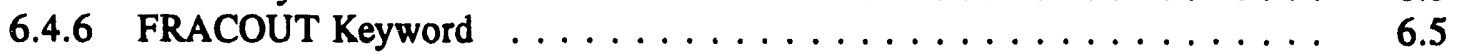

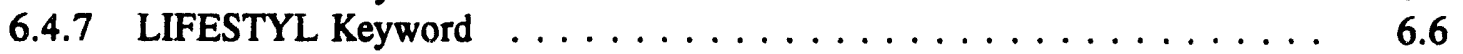

6.4 .8 REALIZAT Keyword $\ldots \ldots \ldots \ldots \ldots \ldots \ldots \ldots \ldots \ldots \ldots$

6.4 .9 REPORT Keyword $\ldots \ldots \ldots \ldots \ldots \ldots \ldots \ldots \ldots \ldots \ldots \ldots$

6.4 .10 SEASON Keyword $\ldots \ldots \ldots \ldots \ldots \ldots \ldots \ldots \ldots \ldots . \ldots \ldots$ 
6.4 .11 SEED Keyword $\ldots \ldots \ldots \ldots \ldots \ldots \ldots \ldots \ldots \ldots \ldots$

6.4 .12 SEX Keyword $\ldots \ldots \ldots \ldots \ldots \ldots \ldots \ldots \ldots \ldots . \ldots \ldots . \ldots \ldots$

6.4 .13 TITLE Keyword $\ldots \ldots \ldots \ldots \ldots \ldots \ldots \ldots \ldots \ldots . \ldots \ldots$

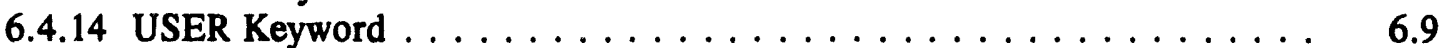

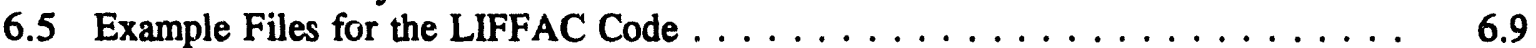

6.5 .1 LIFFAC Keyword File $\ldots \ldots \ldots \ldots \ldots \ldots \ldots \ldots$

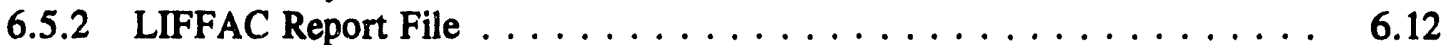

6.5 .3 Lifestyle Factors File $\ldots \ldots \ldots \ldots \ldots \ldots \ldots . \ldots \ldots \ldots$

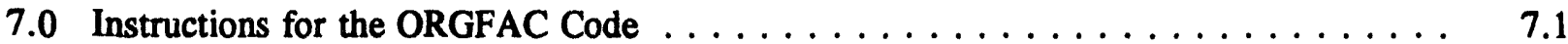

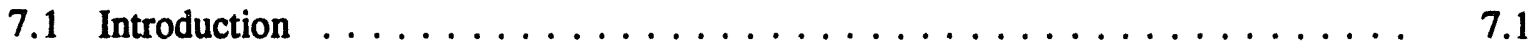

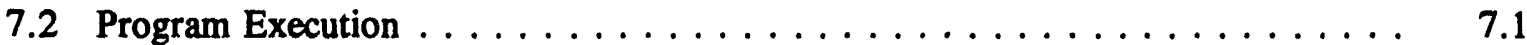

7.2.1 User/System Interface . . . . . . . . . . . . . . . . . . . . 7.1

7.2 .2 Memory Requirements $\ldots \ldots \ldots \ldots \ldots \ldots \ldots \ldots . \ldots \ldots$. . . . . . . .

7.2 .3 Data Size Limitations $\ldots \ldots \ldots \ldots \ldots \ldots \ldots \ldots \ldots . \ldots \ldots . \ldots \ldots$

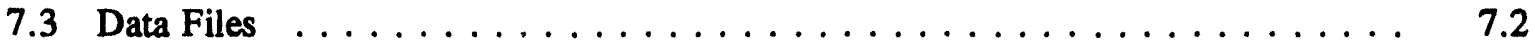

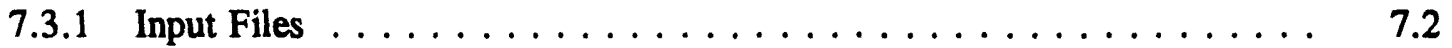

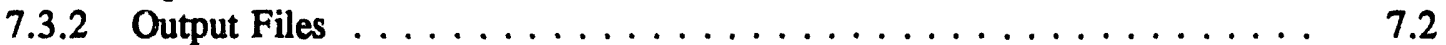

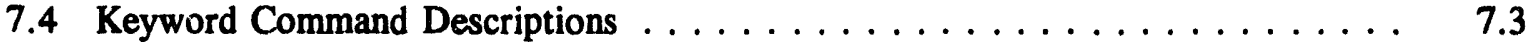

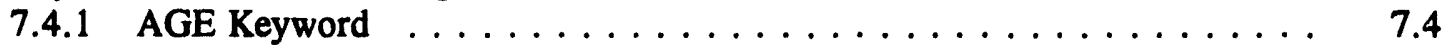

7.4 .2 END Keyword $\ldots \ldots \ldots \ldots \ldots \ldots \ldots \ldots \ldots \ldots \ldots$

7.4 .3 EXTERNAL Keyword $\ldots \ldots \ldots \ldots \ldots \ldots \ldots \ldots \ldots \ldots$

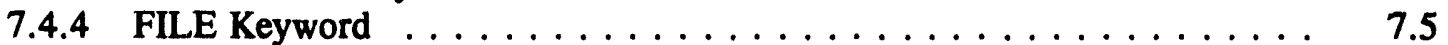

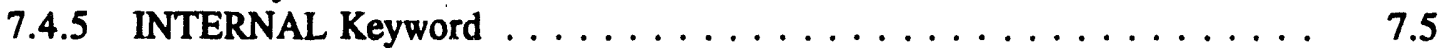

7.4 .6 NUCLIDE Keyword $\ldots \ldots \ldots \ldots \ldots \ldots \ldots \ldots \ldots \ldots$

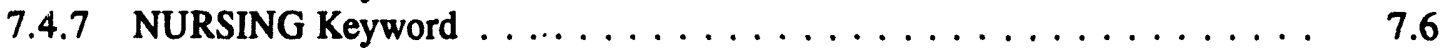

7.4 .8 ORGANS Keyword $\ldots \ldots \ldots \ldots \ldots \ldots \ldots \ldots \ldots \ldots$

7.4 .9 PATH Keyword $\ldots \ldots \ldots \ldots \ldots \ldots \ldots \ldots \ldots \ldots \ldots$

7.4 .10 PRENATAL Keyword $\ldots \ldots \ldots \ldots \ldots \ldots \ldots \ldots \ldots$

7.4 .11 REALIZAT Keyword $\ldots \ldots \ldots \ldots \ldots \ldots \ldots \ldots \ldots \ldots$

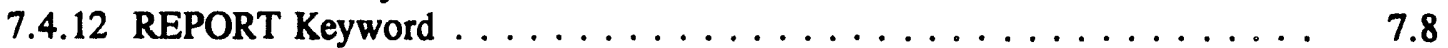

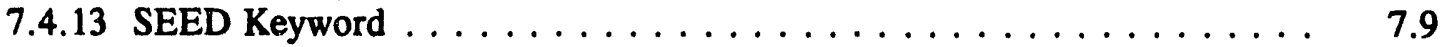

7.4 .14 SEX Keyword . . . . . . . . . . . . . . . . . . . 7.9

7.4 .15 TITLE Keyword $\ldots \ldots \ldots \ldots \ldots \ldots \ldots \ldots \ldots \ldots$

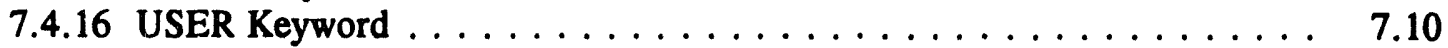

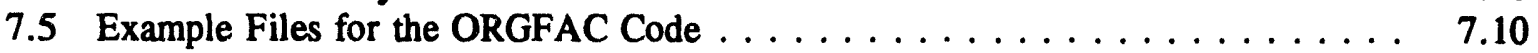

7.5 .1 ORGFAC Keyword File $\ldots \ldots \ldots \ldots \ldots \ldots \ldots \ldots \ldots \ldots$

7.5 .2 ORGFAC Report File $\ldots \ldots \ldots \ldots \ldots \ldots \ldots \ldots \ldots \ldots \ldots$

7.5 .3 Organ Factors File $\ldots \ldots \ldots \ldots \ldots \ldots \ldots \ldots \ldots \ldots \ldots$

8.0 References $\ldots \ldots \ldots \ldots \ldots \ldots \ldots \ldots \ldots \ldots \ldots \ldots \ldots \ldots \ldots$

Appendix A - Text Data File Structure $\ldots \ldots \ldots \ldots \ldots \ldots \ldots \ldots \ldots \ldots \ldots \ldots \ldots$ A.1

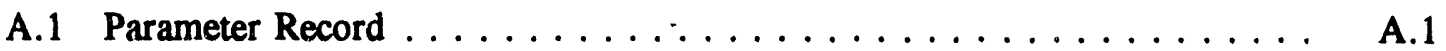

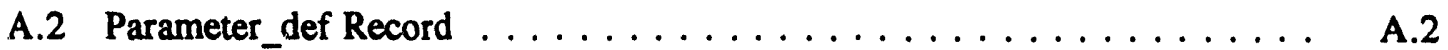

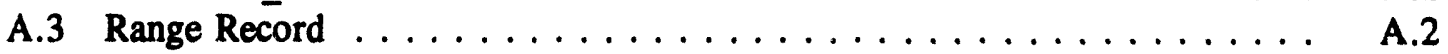

A.4 Data Record . . . . . . . . . . . . . . . . . A.2 
A.4.1 Data Header Record . . . . . . . . . . . . . A.3

A.4.2 Data Specification Record . . . . . . . . . . . . . A.3

A.4.3 Data Record Examples . . . . . . . . . . . . . A.3

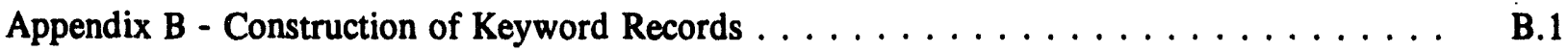

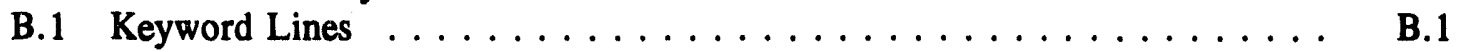

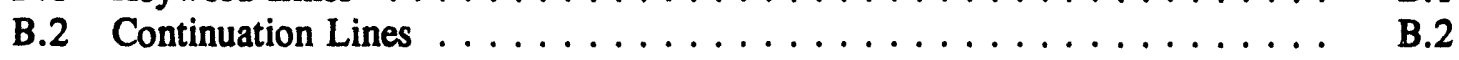

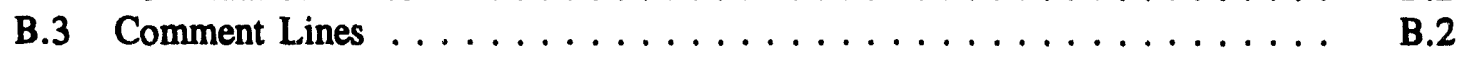

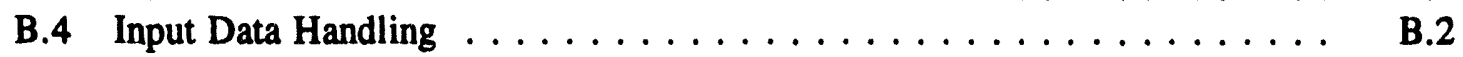

B.5 Quote Strings . . . . . . . . . . . . . . . . . . B.3

B.6 Data Repetition Factors $\ldots \ldots \ldots \ldots \ldots \ldots \ldots \ldots \ldots \ldots \ldots$ B.3

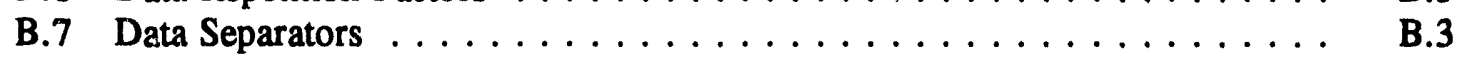

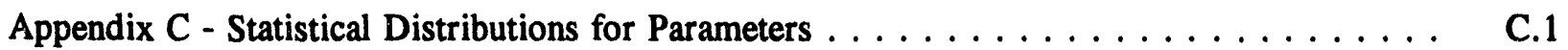

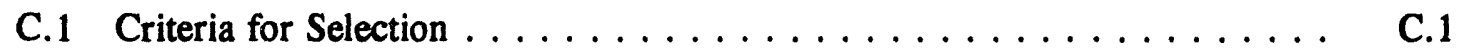

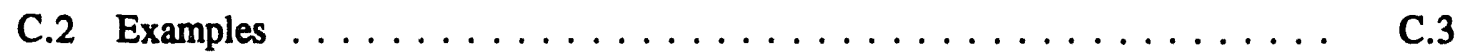

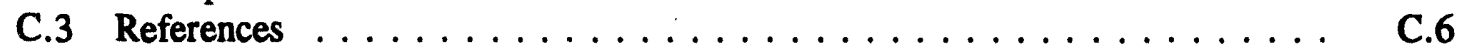




\section{Figures}

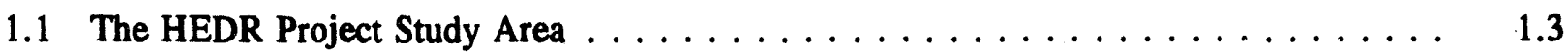

1.2 CIDER Location Information - Modeling Domain $\ldots \ldots \ldots \ldots \ldots$

1.3 Relationship of Codes Supporting CIDER $\ldots \ldots \ldots \ldots \ldots \ldots$

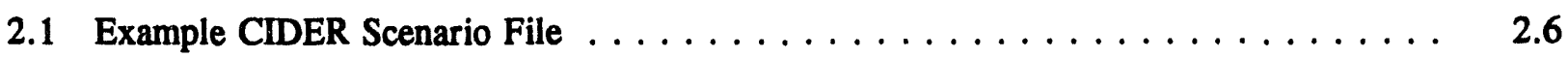

2.2 Example Organ Factors File $\ldots \ldots \ldots \ldots \ldots \ldots \ldots \ldots \ldots \ldots \ldots$

2.3 Example Node Index File $\ldots \ldots \ldots \ldots \ldots \ldots \ldots \ldots \ldots \ldots \ldots \ldots$

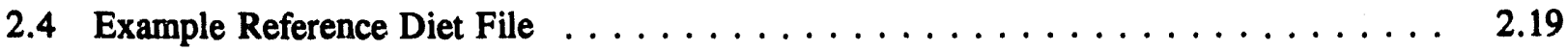

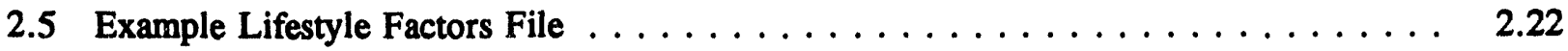

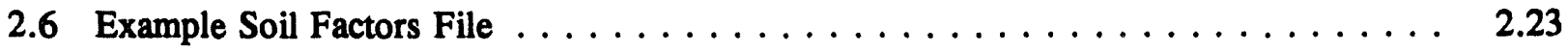

2.7 Example Environmental Factors File $\ldots \ldots \ldots \ldots \ldots \ldots \ldots \ldots \ldots \ldots \ldots$

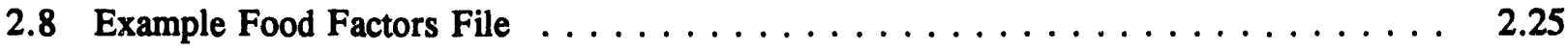

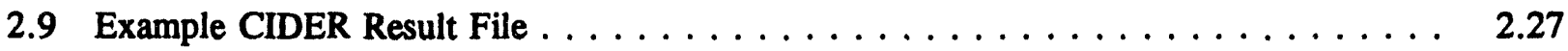

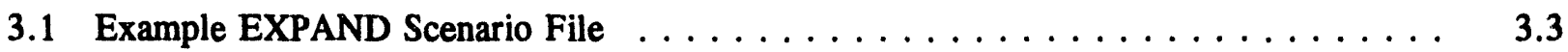

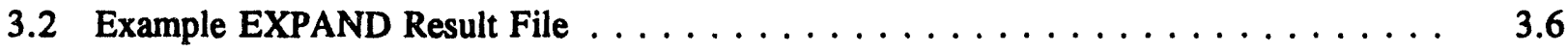

3.3 Example EXPAND Result Key File $\ldots \ldots \ldots \ldots \ldots \ldots \ldots \ldots \ldots$

4.1 Opening Screen from ENVFAC $\ldots \ldots \ldots \ldots \ldots \ldots \ldots \ldots \ldots \ldots \ldots \ldots$

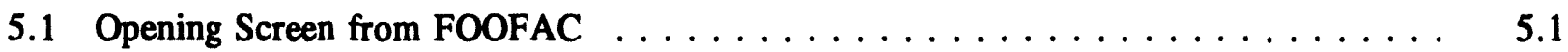

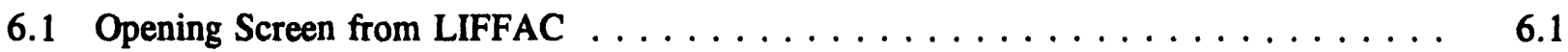

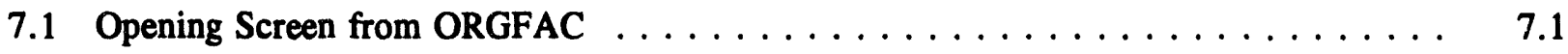




\section{Tables}

1.1 CIDER Data Libraries for Distribution $\ldots \ldots \ldots \ldots \ldots \ldots \ldots \ldots \ldots \ldots \ldots$

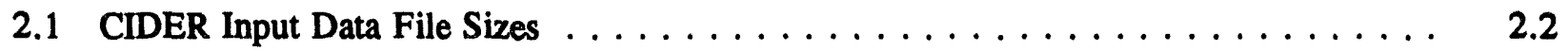

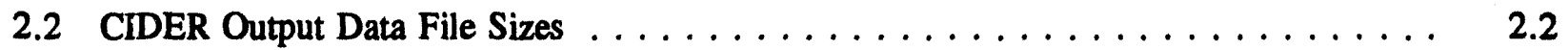

2.3 CIDER Input Files and Their Functions $\ldots \ldots \ldots \ldots \ldots \ldots \ldots \ldots \ldots$

2.4 CIDER Scenario Keyword Summary $\ldots \ldots \ldots \ldots \ldots \ldots \ldots \ldots \ldots \ldots \ldots$

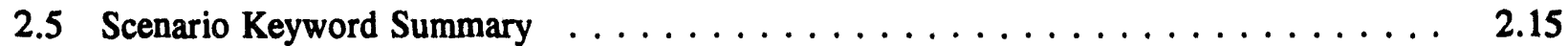

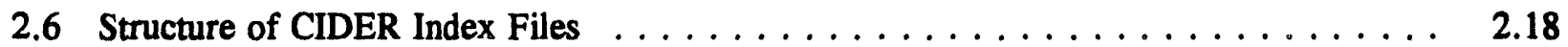

2.7 Structure of Header of the CIDER Result File $\ldots \ldots \ldots \ldots \ldots \ldots \ldots \ldots$

3.1 EXPAND Scenario Keyword Summary $\ldots \ldots \ldots \ldots \ldots \ldots \ldots \ldots \ldots \ldots \ldots$

4.1 ENVFAC Parameter Definitions $\ldots \ldots \ldots \ldots \ldots \ldots \ldots \ldots \ldots \ldots \ldots \ldots \ldots$

4.2 Summary of ENVFAC Keywords $\ldots \ldots \ldots \ldots \ldots \ldots \ldots \ldots$

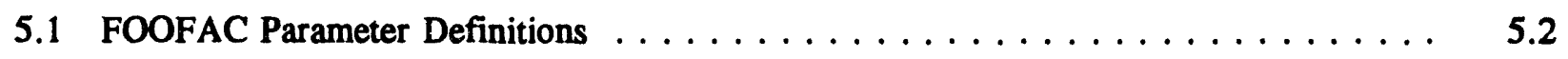

5.2 Summary of FOOFAC Keywords $\ldots \ldots \ldots \ldots \ldots \ldots \ldots \ldots$

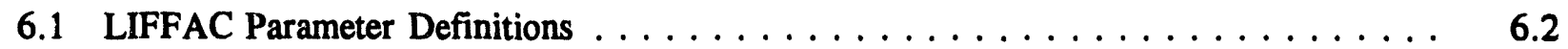

6.2 Summary of LIFFAC Keywords $\ldots \ldots \ldots \ldots \ldots \ldots . \ldots \ldots$

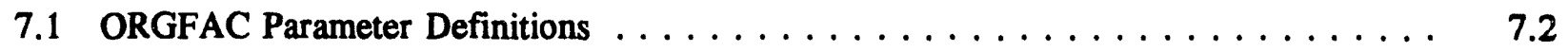

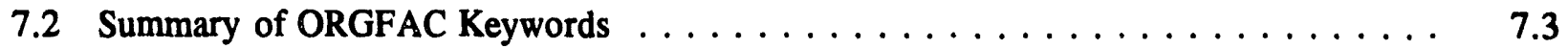




\subsection{Introduetion}

\subsection{Background on HEDR}

The purpose of the HEDR Project is to estimate the radiation dose that individuals could have received as a result of emissions since 1944 from the DOE's Hanford Site near Richland, Washington. The HEDR Project work is conducted under several technical and administrative tasks, among which is the Environmental Pathways and Dose Estimates task. The staff on this task have developed a suite of ccimputer codes which are used to estimate doses to individuals of the public. Dose estimates can be generated with reference input data or with lifestyle, location and consumption data that defines the characteristics of real individuals.

\subsection{Scope of This Document}

This document contains user instruction for the CIDER (Calculation of Individual Doses from Environmental Radionuclides) dose code and five utility codes. The discussion covers the basic structure of the program, the form of the input and output files, the mathematical description of the functions that must be executed, and the major data items.

Four of the utility codes described are used to develop libraries of randomized realizations for model parameters in the CIDER code. These utility prog:ams are

- ENVFAC - environmental factors

- FOOFAC - food factors

- LIFFAC - lifestyle factors, and

- ORGFAC - organ factors.

The final utility is called EXPAND. The EXPAND code processes a result file from CIDER and extracts a summary of the dose information, which could be used as input to separate processes for reporting or plotting.

\subsection{Data File Distribution}

A run of the CIDER code may require up to 10 data libraries. Six of these libraries cannot be modified by the user without destroying the integrity of the data. The other four libraries contain information based on parameter descriptions contained in the HEDR parameter document (Snyder et al, 1992). The ENVFAC, FOOFAC, LIFFAC, and ORGFAC utility programs are provided to allow modification of these libraries. Few, if any, users should have to modify any of the data libraries. The data libraries are described briefly in Table 1.1. The column labelled Modify indicates whether the user is allowed to modify the data library. 
Table 1.1. CIDER Data Libraries for Distribution

\begin{tabular}{|lll||}
\hline Library & Modify & \multicolumn{1}{c|}{ Description } \\
\hline cider.nodes.dat & No & Concentrations of ${ }^{131}$ I in environmental media at each node. \\
cider.nodes.idx & No & Index file for cider.nodes.dat \\
cider.cream.dat & No & $\begin{array}{l}\text { Concentrations of }{ }^{131} \text { I in creamery milk for a set of } 164 \\
\text { creameries }\end{array}$ \\
cider.cream.idx & No & Index file for cider.cream.dat \\
ciderusl.dat & No & Set of mass loadings and soil densities \\
REFERENCE_DIET & No & Reference diets for each age/gender category \\
envfac.dat & Yes & Random samples of environmental parameters \\
foofac.dat & Yes & Random samples of food-related parameters \\
liffac.dat & Yes & Random samples of lifestyle-related parameters \\
orgfac.dat & Yes & Random samples of dose conversions factors for each organ \\
\hline
\end{tabular}

\subsection{Node Locations}

The HEDR modeling domain has been divided into 1102 separate regions, called nodes. Figure 1.1 is a map of the HEDR Project study area. Location information, such as the residence of an individual, is entered in the CIDER code as a node number. Figure 1.2 is provided to assist an individual in locating the node which contains the individual's primary residence.

\subsection{Code Relationships}

This document contains user instructions for the CIDER code and five supporting utility codes. CIDER also receives data from the DESCARTES code as processed by the AGGREGATE code. The relationship between these codes is illustrated in Figure 1.3. Note that the CIDER results file serves as input to the EXPAND code, which generates output information that could be further processed to produce dose reports or plots of dose according to node location.

\subsection{Control Information Relationships}

Control information for all of the codes described in this document is passed into the code through a text file containing keywords. The CIDER and EXPAND codes use keyword records that have very similar syntaxes. The factors programs ENVFAC, FOOFAC, LIFFAC, and ORGFAC all 


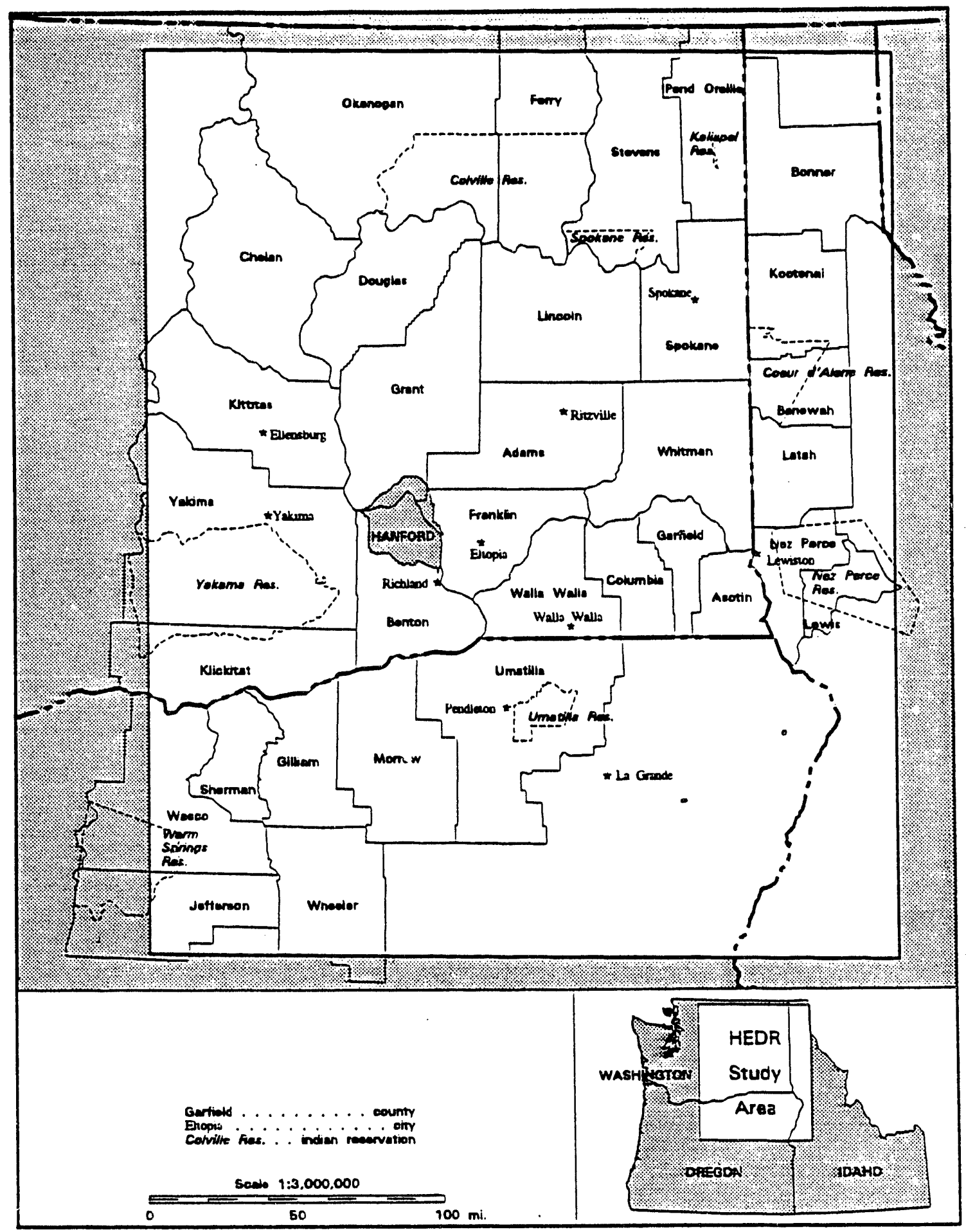

Figure 1.1. The HEDR Project Study Area 


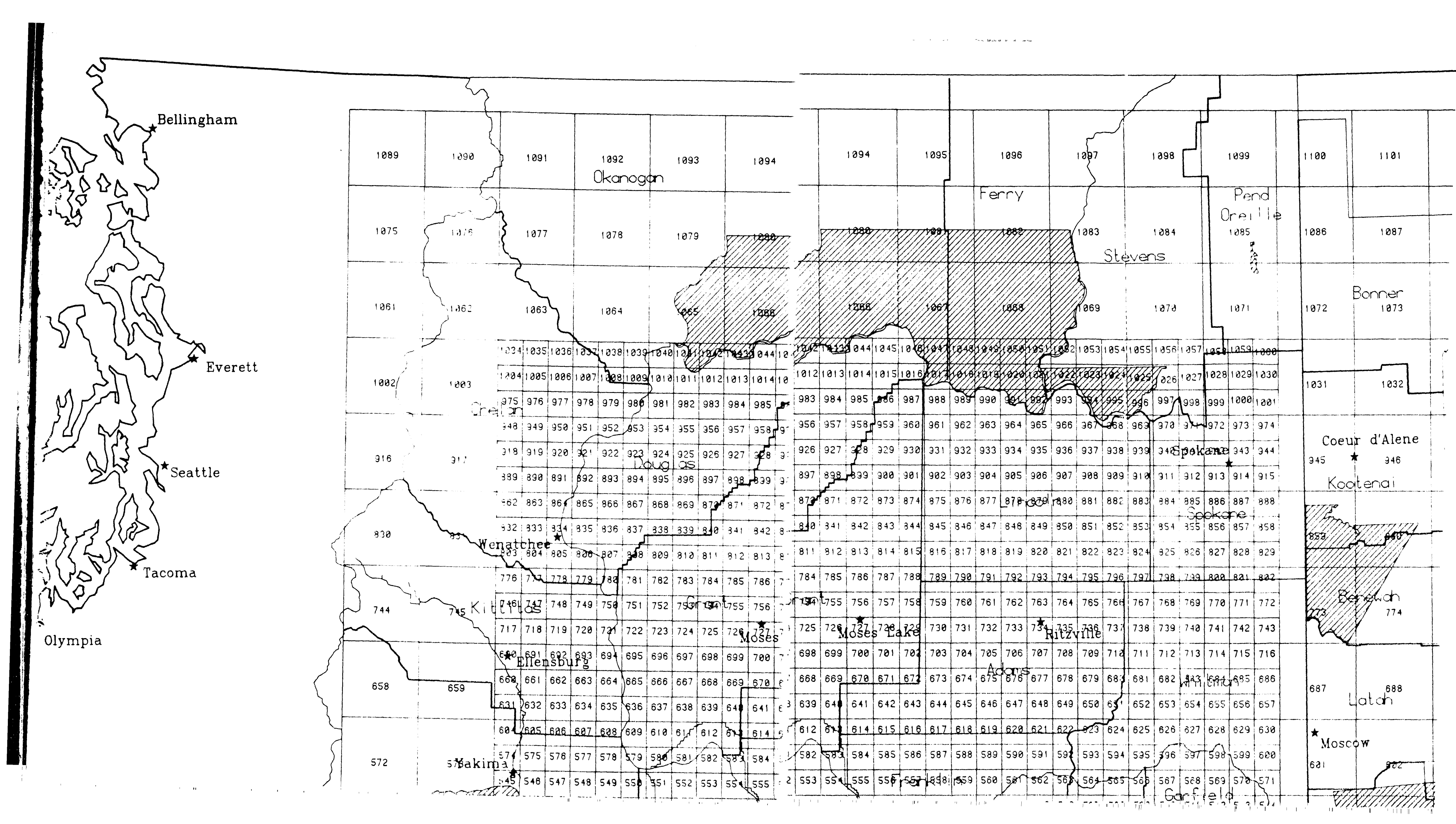




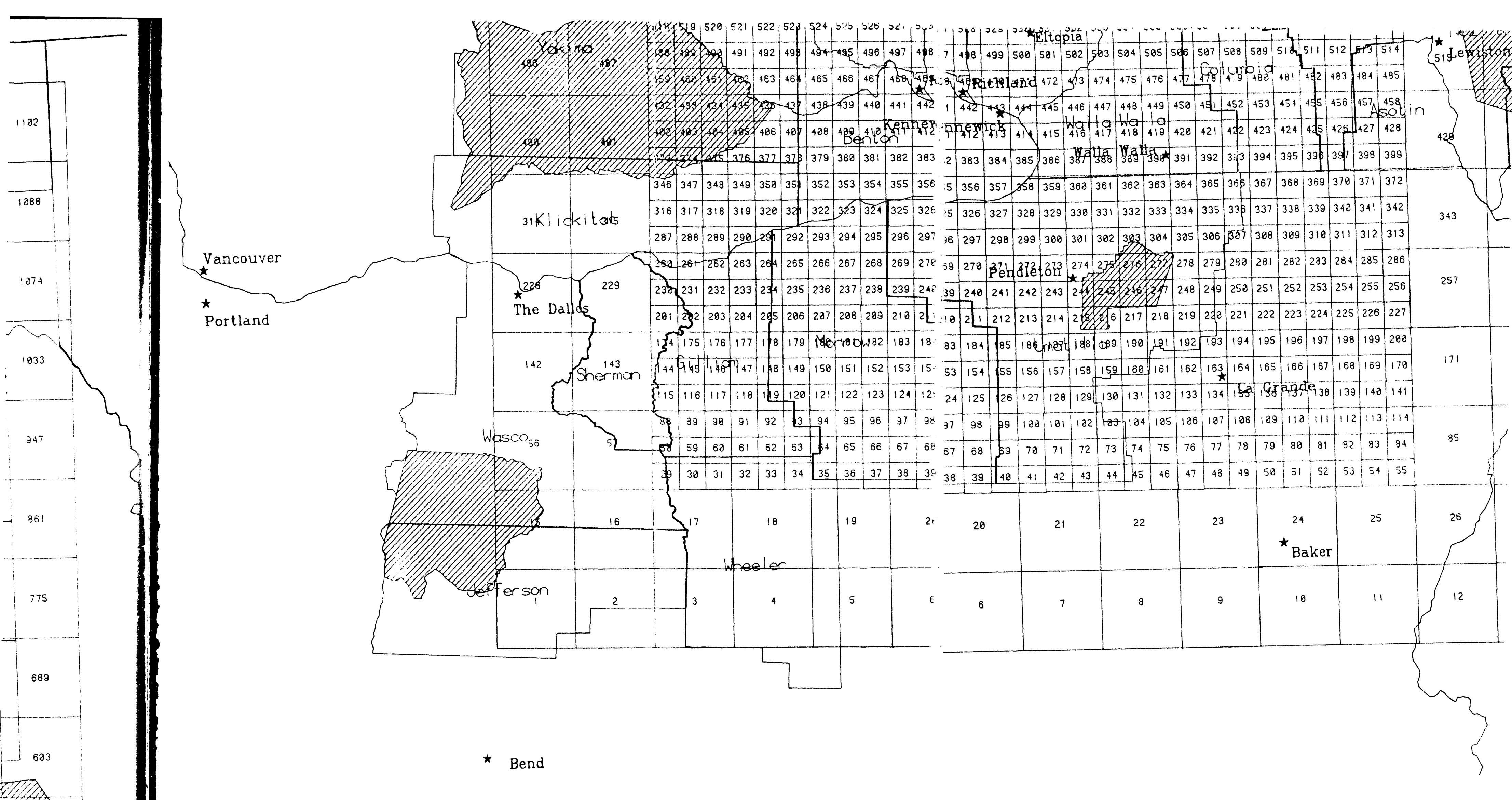




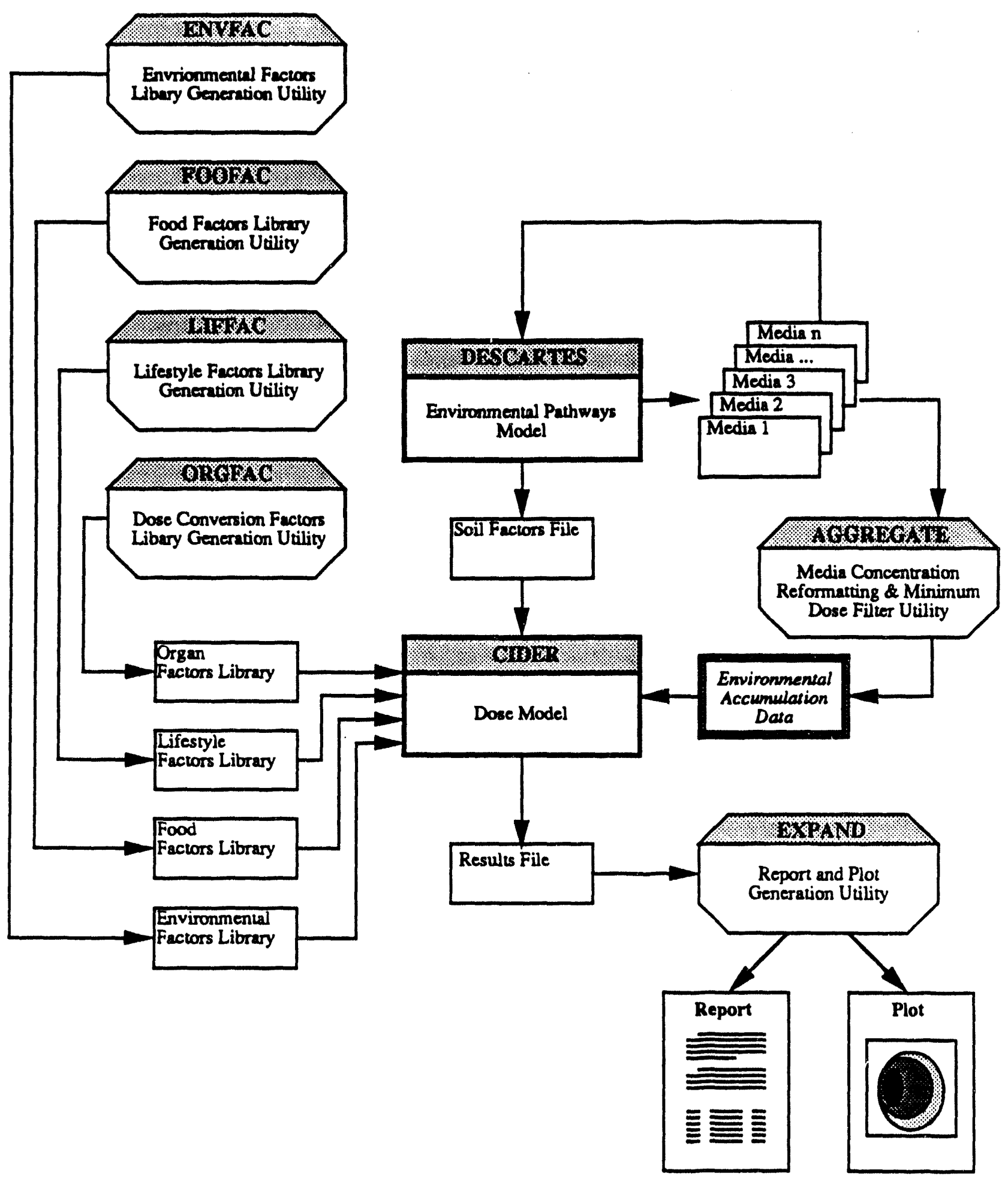

Figure 1.3. Relationship of Codes Supporting CIDER 
very different from that required by the factors programs. The general construction of keywords for CIDER and EXPAND is given in Section 2.2.1. The general construction of keyword records for the factors programs is given in Appendix B.

The CIDER and EXPAND codes also require information from text-based data files. A textbased data file has a special structure. General information concerning the structure of text-based data files is given in Appendix A. The factors programs ENVFAC, FOOFAC, LIFFAC, and ORGFAC read keyword input files and write text-based data files for use in CIDER.

\subsection{Computing Environment}

The HEDR project owns a Sun $4 / 690$. All of the codes described in this document were designed to run on this machine. The HEDR Sun has four processors, 256 megabytes of memory, and 27 gigabytes of disk space. The operating system is SunOS Version 4.1.2 (Unix).

The CIDER and EXPAND codes are written in C and compiled with SPARCompiler $\mathrm{C}++$ version 3.0.1. The ENVFAC, FOOFAC, LIFFAC, and ORGFAC codes are written in FORTRAN 77 and compiled with SC1.0 Fortran Version 1.4. The default compiler flags were used. 


\subsection{Instructions for the CIDER Code}

The CIDER code calculates annual doses to individuals by organ and pathway. The doses are derived from radionuclide concentration estimates provided by the DESCARTES code. CIDER can use assumptions for a reference diet and lifestyle, or the user can specify consumption and lifestyle information.

\subsection{Program Execution}

To calculate a dose, the user will create a file (called the scenario file) that defines the characteristics of the individual(s) for which a dose is to be estimated.

\subsubsection{User/System Interface}

The CIDER code is run by entering the name "cider". The user can provide the names (including a path if desired) for the scenario file and run log file on the command line, or, if this information is omitted, the user will be prompted for the file names. Upon completion of the run, the number of errors and warnings encountered are written to the screen and to the log file. Some examples are:

- To have the code prompt for the scenario and log file names, enter: cider

- To directly supply the scenario and $\log$ file names, enter: cider my_scenario_file.dat my_logfile.dat

- To directly supply the scenario and log file names but run the job in a background mode, enter: cider my_scenario_file.dat my_logfile.dat \&

\subsubsection{Error Processing}

At the conclusion of each run a message concerning the number of warnings and errors generated in the run are written to the screen and to the log file. The error messages and warning messages are written to the log file. All input files are processed to check for errors before any doses are calculated. The execution of CIDER will be aborted:

- if either the scenario or log files can-not be opened

- at the conclusion of the processing of the input data set, if there are any errors

- when the total number of errors and/or warnings exceeds 200 during the processing of the input data

- when any errors are detected during the calculation and reporting of dose values. 


\subsubsection{Memory and Disk Space Requirements}

The CIDER code requires about $32 \mathrm{MB}$ of memory for execution. The code performs dynamic memory allocation, so different runs may use different amounts of memory.

The CIDER code requires a large database of information in order to operate. Table 2.1 gives a summary of the disk space required for input files to CIDER. Table 2.2 gives a summary of output file sizes for several uses of CIDER. All of the output files are based on runs requesting 100 realizations of dose estimates.

Table 2.1. CIDER Input Data File Sizes (megabytes)

\begin{tabular}{||rl||}
\hline File Size, MB & Description \\
\hline 11 & Concentrations for milk from creameries \\
995 & Concentrations from all media except creamery milk \\
$<1$ & Stochastic parameter libraries \\
1 & Reference diet library \\
$<1$ & Scenario file \\
1,007 & Total \\
\hline
\end{tabular}

Table 2.2. CIDER Output Data File Sizes (kilobytes)

\begin{tabular}{|rl|}
\hline File Size, KB & Description \\
\hline 13.7 & Dose to an individual for 1945 \\
160.8 & Dose to an individual for $1944-1957$ \\
13,500 & Doses for a map run on 1102 nodes for 1945 \\
101,200 & Doses for a map run on 1102 nodes for $1944-1957$ \\
\hline
\end{tabular}

\subsection{Input File Specifications}

Input files to the CIDER code consist of a command file (hereafter called the scenario file) and multiple data files. The user/analyst will be responsible for the generation of the scenario file. The other data files will be provided with the code. It is not expected that the casual user will ever need to modify any file except the scenario file. The CIDER input files and their functions are summarized in Table 2.3. For completeness, the contents and syntax of the additional files are also described. 
Table 2.3. CIDER Input Files and Their Functions

\begin{tabular}{|c|c|}
\hline Fle & Function \\
\hline scenario & $\begin{array}{l}\text { The scenario file contains all information needed to define the characteristics of the individuals } \\
\text { for which doses are to be calculated, and the names and locations of all other input and output } \\
\text { files required by the code. The name and location of the scenario file are provided on the } \\
\text { command line when starting CIDER, or are supplied in response to a prompt if omitted from the } \\
\text { command line. The file is in text format and is always required. This file is generated by the } \\
\text { user. }\end{array}$ \\
\hline node concentration & $\begin{array}{l}\text { The node concentration file(s) contain the temporal environmental contaminant levels for a } \\
\text { specific radionuclide and all media except creameries. The nanke(s) and location(s) of the node } \\
\text { concentration file(s) are contained in the node index file identified by the "LIB_ENV_NODE" } \\
\text { keyword in the scenario file. The file is in binary format and is always required. This file is } \\
\text { generated by the AGGREGATE code. }\end{array}$ \\
\hline $\begin{array}{l}\text { creamery } \\
\text { concentration }\end{array}$ & $\begin{array}{l}\text { The creamery concentration file(s) contain the temporal contaminant levels for a specific } \\
\text { radionuclide and all creameries. The name(s) and location(s) of the creamery concentration } \\
\text { file(s) are contained in the creamery index file identified by the "LIB_ENV_CREAM" keyword } \\
\text { in the scenario file. The file is in binary format and is required only if milk from creameries is } \\
\text { opecified in a diet. This file is generated by the AGGREATE code. }\end{array}$ \\
\hline node index & $\begin{array}{l}\text { The node index file contains the information needed to locate data for a given year and node in } \\
\text { the node concentration data file(s). The node index file name and location are identified with } \\
\text { the "LIB_ENV_NODE" keyword in the scenario file. The file is in text format and is always } \\
\text { required. This file is generated by the AGGREGATE code. }\end{array}$ \\
\hline creamery index & $\begin{array}{l}\text { The creamery index file contains the information needed to locate data for a given year and } \\
\text { creamery in the creamery concentration data file(s). The creamery index file name and location } \\
\text { are identified by the "LIB_ENV_CREAM" keyword in the scenario file. The file is in text } \\
\text { format and is required only if creamery milk is specified in the diet. This file is generated by } \\
\text { the AGGREGATE code. }\end{array}$ \\
\hline organ factors & $\begin{array}{l}\text { The organ factors file contains the information needed to specify the dose factors for each } \\
\text { pathway, organ, and nuclide. The organ factors file name and location are identified by the } \\
\text { "LIB_ORGAN_FACTORS" keyword in the scenario file. The file is in text format and is } \\
\text { always required. This file is generated by the ORGFAC code. }\end{array}$ \\
\hline soil factor & $\begin{array}{l}\text { The soil factor file contains the ratio of mass loading to upper soil density (ML/tho ual) that was } \\
\text { used in the DESCARTES code to generate the media concentration estimates. The mame and } \\
\text { version of the soil factor files are contained in the node and creamery index files. The soil } \\
\text { factors file is in text format and is always required. This file is provided from DESCARTES } \\
\text { output. }\end{array}$ \\
\hline $\begin{array}{l}\text { environmental } \\
\text { factors }\end{array}$ & $\begin{array}{l}\text { The environmental factors file contains information on ground shielding, and the ratio of time } \\
\text { spent indoors to time spent in outdoor activity. The environmental factors file name and } \\
\text { location are identified by the "LIB_ENV_FACTORS" keyword in the scenario file. The file is } \\
\text { in text format and is always required. This file is generated by the ENVFAC code. }\end{array}$ \\
\hline reference diet & $\begin{array}{l}\text { The reference diet file contains the diet information for each reference individual. The reference } \\
\text { diet file name and location are identified by the "LIB_REF_DIET" keyword in the scenario file. } \\
\text { The file is in text format and is always required. }\end{array}$ \\
\hline lifestyle & $\begin{array}{l}\text { The lifestyle file contains the breathing rate and fraction of day spent outdoors information. The } \\
\text { lifestyle file name and location are identified by the "LIB_LIFESTYLE" keyword in the scenario } \\
\text { file. The file is in text format and is always required. This file is generated by the LIFFAC } \\
\text { code. }\end{array}$ \\
\hline food factors & $\begin{array}{l}\text { The food factors file contains information on holdup times, dry-to-wet conversion factors, and } \\
\text { retention fractions for washed fruit and vegetables. The food factors file name and location are } \\
\text { identified by the "LIB_FOOD_FACTORS" keyword in the scenario file. The file is in text } \\
\text { format and is always required. This file is generated by the FOOFAC code. }\end{array}$ \\
\hline
\end{tabular}


If the user chooses to modify any file other the scenario file, it is incumbent on the user to understand all the implications of the change. The four utility programs ENVFAC, FOOFAC, LIFFAC, and ORGFAC are also described in this document. These utility codes can be used to generate four input data libraries for the CIDER code.

\subsubsection{Scenario File Definition}

The scenario file is a text file generated by the user that defines the characteristics of the case individuals being modeled and the source of the input data. This file uses keyword-driven records. The general rules used to construct the scenario text files are given here. A more extended discussion of the format of a text file is given in Appendix A.

- Each record is terminated by a semicolon (;).

- A record may cover one or more lines but no more than one record per line is allowed.

- Comments may be placed anywhere in the file between a semicolon (;) and an end of line $(<$ eol $>)$.

- Case is not significant for keywords.

- Fields are delimited by any non-valid token character (such as a space, comma, or tab-see the glossary)

- Unless noted, leading and trailing spaces are acceptable.

- The length of a line is limited only by the operating system.

- A token is no longer than 80 characters.

- A null record (a record that begins with a terminating semicolon) is allowed (for example, '; this is a comment').

The scenario file contains two types of data: general data and case specific data. The general data is placed at the beginning of the file and applies to all the cases within a given scenario file (for example, names and locations of the input and output files, numbers of realizations). The casespecific data defines the characteristics of an individual whose dose is to be calculated (for example, age, sex, lifestyle, diet, residence locations).

Unless noted otherwise, only one specification of each keyword is allowed in a scenario file. If such a keyword is specified multiple times, the last one in the list will be used. If multiple instances of the same type of data file are specified, the data loaded are cumulative with the later data overwriting the earlier data.

The keywords used in the scenario file are summarized in Table 2.4. The column labeled "CASE" specifies whether the keyword applies to the case definition $(\mathrm{Y})$ or general data $(\mathrm{N})$. The 
column labeled "REQ" indicates whether or not the keyword must be provided. The column labeled "DEFAULT" indicates whether there is a default and, if so, the value of the default. More detailed descriptions of the scenario keywords are given in the following sections.

Table 2.4. CIDER Scenario Keyword Summary

\begin{tabular}{|c|c|c|c|}
\hline KEYWORD & CASE & REQ & DEFAULT \\
\hline ABORT & $\overline{\mathbf{N}}$ & $\overline{\mathbf{N}}$ & No aborting \\
\hline ANALYST & $\mathbf{N}$ & $\mathbf{N}$ & "unknown" \\
\hline BEGIN & $\mathbf{Y}$ & $\mathbf{Y}$ & None \\
\hline BIRTH & $\mathbf{Y}$ & $\mathbf{Y}$ & None \\
\hline DIET & $\mathbf{Y}$ & $\mathbf{N}$ & $\begin{array}{l}\text { Reference diet selection based on } \\
\text { individual characteristics }\end{array}$ \\
\hline DIET_SPEC & $\mathbf{N}$ & $\mathbf{N}$ & None \\
\hline END & $\mathbf{Y}$ & $\mathbf{Y}$ & None \\
\hline FINISH & $\mathbf{Y}$ & $\mathbf{Y}$ & None \\
\hline LIB_ENV_CREAM & $\mathbf{N}$ & $\mathbf{N}$ & None \\
\hline LIB_ENV_NODE & $\mathbf{N}$ & $\mathbf{Y}$ & None \\
\hline LIB_FOOD_FACTOR & $\mathbf{N}$ & $\mathbf{Y}$ & None \\
\hline LIB_LIFESTYLE & $\mathbf{N}$ & $\mathbf{Y}$ & None \\
\hline LIB_ORGAN_FACTOR & $\mathbf{N}$ & $\mathbf{Y}$ & None \\
\hline LIB_REF_DIET & $\mathbf{N}$ & $\mathbf{Y}$ & None \\
\hline LIB_ENV_FACTOR & $\mathbf{N}$ & $\mathbf{Y}$ & None \\
\hline LIFESTYLE & $\mathbf{Y}$ & $\mathbf{N}$ & Urban \\
\hline LOC & $\mathbf{Y}$ & $\mathbf{Y}$ & $\begin{array}{l}\text { None, required only if MAP is } \\
\text { undefined }\end{array}$ \\
\hline MAP & $\mathbf{Y}$ & $\mathbf{Y}$ & $\begin{array}{l}\text { None, required only if LOC is } \\
\text { undefined }\end{array}$ \\
\hline MOTHER_BIRTH & $\mathbf{Y}$ & $\mathbf{N}$ & Age 25 at birth of case individual \\
\hline MOTHER_DIET & $\mathbf{Y}$ & $\mathbf{N}$ & $\begin{array}{l}\text { Reference diet for pregnant or } \\
\text { nursing female }\end{array}$ \\
\hline ORGANS & $\mathbf{N}$ & $\mathbf{N}$ & Thyroid \\
\hline PATHWAYS & $\mathbf{N}$ & $\mathbf{N}$ & All pathways \\
\hline PREG_NURSE & $\mathbf{Y}$ & $\mathbf{N}$ & Never pregnant or nursing \\
\hline REALIZATIONS & $\mathbf{N}$ & $\mathbf{N}$ & 100 \\
\hline RESULT_FILE & $\mathbf{N}$ & $\mathbf{Y}$ & None \\
\hline SEX & $\mathbf{Y}$ & $\mathbf{N}$ & None \\
\hline START & $\mathbf{Y}$ & $\mathbf{Y}$ & None \\
\hline WASHED & $\mathbf{Y}$ & $\mathbf{N}$ & Yes, washed \\
\hline WEANED & $\mathbf{Y}$ & $\mathbf{N}$ & Birth date \\
\hline
\end{tabular}

An example CIDER scenario file is given in Figure 2.1. 


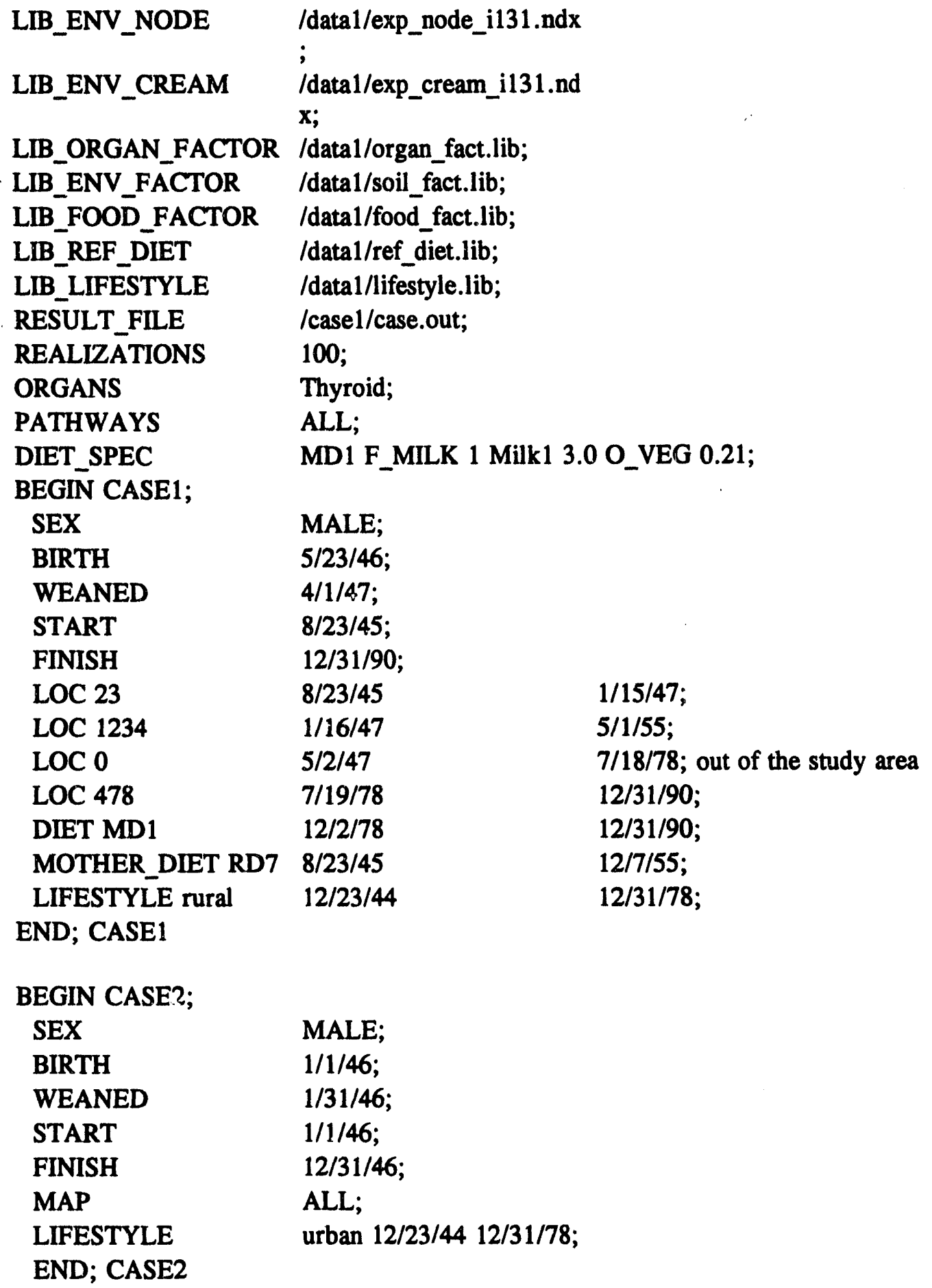

SEX

BIRTH

WEANED

START

FINISH

MAP

LIFESTYLE

END; CASE2

MALE;

1/1/46;

1/31/46;

$1 / 1 / 46$;

12/31/46;

ALL;

urban 12/23/44 12/31/78;

Figure 2.1. Example CIDER Scenario File 


\subsubsection{ABORT Keyword}

The ABORT record forces the termination of the run after the first error is detected in the processing of the input data. An example entry is the following:

\section{ABORT;}

\subsubsection{ANALYST Keyword}

The ANALYST record specifies the name of the analyst that constructed the scenario file. The form of the name must conform to the rules used to form a token (see the glossary). If this record is omitted, the analyst name will default to "unknown." The analyst name will be echoed to the result file. An example record is the following:

ANALYST J_Smith;

\subsubsection{BEGIN Keyword}

The BEGIN record denotes the start of a case definition. A token uniquely identifying the case must follow the keyword. The case identification is written as part of the dose records in the result file. No defaults are provided for this record. A case definition is terminated by an END record and each BEGIN record must always be paired with an END record. At least one case must be specified in the scenario file. Up to CASE (see Section 2.5.3) cases may be defined in a single scenario file. The following are some of the keywords that may be specified in a case definition: sex, birth, weaned, start, end, loc, map, diet, and lifestyle. An example record is the following:

BEGIN case23a;

\subsubsection{BIRTH Keyword}

The BIRTH record denotes the birth date of the case individual. The form of the date record is "mm/dd/yy". This record is required. No default is provided. If the birth date is prior to 1900 , use " 00 " for the year. An example record is the following:

BIRTH 1/23/45;

\subsubsection{DIET Keyword}

The DIET record is used to specify the diet of the individual. Diets records contain a date range for which they are to be applied. Multiple diet specifications are allowed. Multiple DIET records are required to define a user-specified diet that changes with time. The names of the reference diets are contained in the diet file. The following items are specified as part of this record:

1) Diet identification - This must be a reference diet identification or a diet defined by a DIET_SPEC record. 
2) Starting date $(\mathrm{mm} / \mathrm{dd} / \mathrm{yy})$ - inclusive

3) Ending date $(\mathrm{mm} / \mathrm{dd} / \mathrm{yy})$ - inclusive.

If no diet information is provided, a default diet will be selected based upon the characteristics of the case individual. The default diet is described by Anderson, Bates, and Marsh (1993). DIET records (see also DIET_SPEC records) should be specified only if there is objective information on the consumption patterns of the subject individual.

An example DIET record entry is the following:

DIET RD1 1/2/46 1/7/48;

\subsubsection{DIET_SPEC Keyword}

The DIET_SPEC record is used to allow the user to define a special diet category for use in one or more cases. No scaling of DIET_SPEC diets is applied. (Reference diets are derived from 1977 data. This data is converted to diets for the period of 1944 to 1951 by a time dependent scaling factor (gaf_annual_scaling_factor) entered in the reference diet library). (Anderson et al. 1993). The units for food are $\mathrm{kg}$ per day for all foods except milk, which has units of $1 /$ day. The following items are specified as part of the diet record:

1) Diet identification - This must be a unique token identifying this diet. It is not allowable to redefine a reference diet.

The following records may be repeated until all changes are stated.

2a) Name of a food type

If field 2a is fresh milk (F_MILK) or stored milk (S_MILK), then field $2 b$ is the milk order number (1 through 5) and field $2 c$ must specify the type of milk as a milk media symbol (for example, milk_2), or if creamery milk is used, field $2 \mathrm{~b}$ must specify the creamery id number. When specifying a milk food (fresh or stored), all 5 milks must be specified even if the consumption is zero.

If field $2 a$ is leafy vegetables (L_VEG), field $2 b$ must specify the fraction of leafy vegetables consumed that are local (for example, 0.43). Otherwise this field is skipped.

2b) Modifier of the food type (see preceding note)

2c) Secondary modifier of food type (see preceding note)

3) Quantity of food provided from the study area consumed per day in kilograms (kg) or liters (L). Do not specify a count such as a number of bananas when specifying fruit. The entry must be an exact value (such as 0.45 ) 
The foods that are currently valid are specified in the FOODTYPE range parameter (see Section 2.5.3). An example record is the following:

DIET_SPEC MD4a S_MILK 1 MILK_1 .45 S_MILK 212.13 S_MILK 3 MILK_1 0.00 S_MILK 4 milk_2 0.00 S_MILK 5 milk_3 0.00 O_VEG 25 FRUIT 1.5 L_VEG .25 .76 beef .45 ;

The DIET_SPEC record is optional; reference diets are provided as defaults. The DIET_SPEC specification overrides only the food types explicitly identified. Any food types not specified will default to the reference diet values. Up to DIET_ID (see Section 2.5.3) diets may be specified (including the reference diets).

\subsubsection{END Keyword}

The END keyword denotes the end of a case definition (see the BEGIN keyword description). The END record is always paired with a BEGIN record. An example record is the following:

END;

\subsubsection{FINISH Keyword}

The FINISH record specifies the date (inclusive) to stop the calculation of doses for the given case. This record must be paired with a START record within a case definition. This record is required. An example record is the following:

FINISH 5/30/54;

\subsubsection{LB_ENV_CREAM Keyword}

The LIB_ENV_CREAM record is used to identify the name and location of the index file associated with the creamery concentration data. This record is required only if a DIET_SPEC diet includes milk from creameries. No defaults are provided. An example record is the following:

LIB_ENV_CREAM/data1/exp_cream_i131.ndx;

\subsubsection{LIB_ENV_NODE Keyword}

The LIB_ENV_NODE record is used to identify the name and location of the index file associated with the node concentration data. There is no default for this record, and it must be specified. An example record is the following:

LIB_ENV_NODE /data1/exp_node_i131.ndx; 


\subsubsection{LIB_FOOD_FACTOR Keyword}

The LIB_FOOD_FACTOR record is used to identify the name and location of the file containing the food factors library. There is no default for this record, and it must be specified. An example record is the following:

LIB_FOOD_FACTOR /data1/food_factor_i131.lib;

\subsubsection{LIB_LIFESTYLE Scenario Keyword}

The LIB_LIFESTYLE record is used to identify the name and location of the file containing the lifestyle factors library. There is no default for this record, and it must be specified. An example record is the following:

LIB_LIFESTYLE /data1/lifestyle.lib;

\subsubsection{LIB_ORGAN_FACTOR Keyword}

The LIB_ORGAN_FACTOR record is used to identify the name and location of the file containing the organ factors library. There is no default for this record, and it must be specified. An example record is the following:

LIB_ORGAN_FACTOR /data1/org_factor_i131.lib;

\subsubsection{LIB_REF_DIET Keyword}

The LIB_REF_DIET record is used to identify the name and location of the file containing the reference diet library. There is no default for this record, and it must be specified. An example record is the following:

\section{LIB_REF_DIET /data1/ref_diet.lib;}

\subsubsection{LIB_ENV_FACTOR Keyword}

The LIB_ENV_FACTOR record is used to identify the name and location of the file containing the environmental factors library. There is no default for this record, and it must be specified. An example record is the following:

LIB_ENV_FACTOR/data1/env_factor.lib;

\subsubsection{LIFESTYLE Keyword}

The LIFESTYLE record is used to specify the lifestyle of the individual. Lifestyles are specified by date. Multiple LIFESTYLE records are required to define a lifestyle change. The following items are specified as part of this record: 
1) Lifestyle identification - This must be either "rural" or "urban" (see LIFESTYLE in Section 2.5.3).

2) Starting date $(\mathrm{mm} / \mathrm{dd} / \mathrm{yy})$ - inclusive

3) Ending date (mm/dd/yy) - inclusive.

The lifestyle will default to urban for any time period not specified. If the case involves a prenatal or nursing infant, the mother's lifestyle is assumed to be the same as the child's lifestyle. An example record is the following:

\section{LIFESTYLE URBAN 1/2/46 1/7/48;}

\subsubsection{LOC Keyword}

The LOC record is used to specify the residence locations for the individual during the dose study period. Residence locations are specified as a node number. Node numbers range from 1 to 1102 in the base data set (see Section 2.5.3). Each LOC record defines a location for a given period. Multiple LOC records are required if the individual changes residence locations. Time periods may overlap with later records taking precedence over earlier records. Locations are specified by date. Node zero ( 0 ) is used to indicate that the individual is out of the study area for a period of time. The following items are specified as part of this record:

1) Node identification - A node identification of zero ( 0 ) indicates that the individual is outside the area of interest during that period

2) Starting date $(\mathrm{mm} / \mathrm{dd} / \mathrm{yy})$ - inclusive

3) Ending date $(\mathrm{mm} / \mathrm{dd} / \mathrm{yy})$ - inclusive

If LOC records are used, locations must be specified for the entire period of the study. A scenario file may contain both LOC and MAP records but a case must only have a LOC or MAP record, but not both. Example entries are:

LOC $231 / 2 / 461 / 7 / 48$; two years at node 23

LOC 0 6/1/45 10/23/45; out of area for 4 months

\subsubsection{MAP Keyword}

The MAP record is used to indicate a case where the same individual is placed in many locations simultaneously during the period of study to construct a dose map. The keyword MAP may be followed by a list of nodes of interest or the word "ALL" (indicates that all nodes are to be calculated. If nothing follows the keyword MAP, it is assumed to be "ALL". The LOC record cannot be used in the same case as a MAP record. If multiple MAP records are provided, they are additive. Example entries are: 
MAP 12345 ;

MAP all;

MAP;

\subsubsection{MOTHER_BIRTH Keyword}

The MOTHER_BIRTH record denotes the birth date of the mother of the case individual. The form of the date record is "mm/dd/yy". This record is optional. The default is the birth date of the case individual less twenty five (25) years. If the mother's birth is prior to 1900 , use " 00 " for the year. An example record is the following:

MCTHER_BIRTH 1/23/20;

\subsubsection{MOTHER_DIET Keyword}

The MOTHER_DIET record is used to define the diet of the mother of a prenatal or nursing child. The format for this record is the same as the DIET record. Multiple MOTHER_DIET diet specifications are allowed. Multiple MOTHER_DIET records are required to define a user-specified diet that changes with time. The names of the reference diets are contained in the reference diet file. The following items are specified as part of this record:

1) Diet identification - This must be a reference diet identification or a diet defined by a DIET_SPEC record.

2) Starting date $(\mathrm{mm} / \mathrm{dd} / \mathrm{yy})$ - inclusive

3) Ending date $(\mathrm{mm} / \mathrm{dd} / \mathrm{yy})$ - inclusive.

If no diet information is provided, a default diet will be selected based upon an assumed mother's age of 25 at the birth of the case individual. The default diet is described by Anderson, Bates, and Marsh (1993). MOTHER_DIET records (see also DIET_SPEC records) should be specified only if there is objective information on the consumption patterns of the subject individual. An example record is the following:

MOTHER_DIET RD1 1/2/46 1/7/48;

\subsubsection{ORGANS Keyword}

The ORGANS record is used to identify or limit the organs for which doses are calculated. If this record is omitted, it will default to using the thyroid. The current list of valid organs is the following: thyroid and whole_body (see the parameter ORGANS in Section 2.5.3). An example record is the following:

ORGANS thyroid; 